\title{
Superfluidity in a Model of Massless Fermions Coupled to Scalar Bosons
}

\author{
Robert D. Pisarski ${ }^{1}$ and Dirk H. Rischke ${ }^{2}$ \\ ${ }^{1}$ Department of Physics \\ Brookhaven National Laboratory, Upton, New York 11973, U.S.A. \\ email: pisarski@bnl.gov \\ 2 RIKEN-BNL Research Center \\ Brookhaven National Laboratory, Upton, New York 11973, U.S.A. \\ email: rischke@bnl.gov
}

\begin{abstract}
We study superfluidity in a model of massless fermions coupled to a massive scalar field through a Yukawa interaction. Gap equations for a condensate with total spin $J=0$ are solved in the mean-field approximation. For the Yukawa interaction, the gaps for rightand left-handed fermions are equal in magnitude and opposite in sign, so that condensation occurs in the $J^{P}=0^{+}$channel. At finite scalar mass, there are two different gaps for fermions of a given chirality, corresponding to condensation of particle pairs or of antiparticle pairs. These gaps become degenerate in the limit of infinite scalar mass.
\end{abstract}

\section{INTRODUCTION}

In fermionic matter which is sufficiently cold and dense, any attractive interaction at the Fermi surface leads to the formation of Cooper pairs [1]. The Cooper pairs form a Bose condensate, so that exciting a quasiparticle costs an amount of energy $\geq 2|\phi|$, where $|\phi|$ is the gap energy. This gap produces superfluidity, or, if the fermions are coupled to a gauge field, superconductivity.

In quantum chromodynamics (QCD), one-gluon exchange between two quarks is attractive in the colorantitriplet channel. One therefore expects that at sufficiently large quark chemical potential $\mu$ and sufficiently small temperature $T$ quarks condense into Cooper pairs which are color antitriplets. This condensate breaks the $S U(3)$ color symmetry of the ground state, and gives rise to color superconductivity. For QCD, this phenomenon was first investigated by Barrois [2], by Bailin and Love [3], and others [4]. In seminal work, Bailin and Love estimated the gap energy to be on the order of $|\phi| \sim 10^{-3} \mu$. Since the critical temperature for the onset of superconductivity, $T_{c}$, is of the order of the gap energy, $|\phi|$, a color-superconducting phase of quark matter could form in the interior of neutron stars. Color superconductivity was also studied in an $S U(2)$ gauge theory $[5]$.

Interest in this subject was renewed through works by Alford, Rajagopal, and Wilczek [6] and by Rapp, Schäfer, Shuryak, and Velkovsky [7]. In these papers, the attractive interaction was modeled with the instanton vertex. In the framework of a simple model of the type studied by Nambu and Jona-Lasinio (NJL) [8], these authors found gap energies of the order of $|\phi| \sim \mu$. Since $T_{c} \sim|\phi|$, for gap energies of this order of magnitude color superconductivity is no longer only relevant for astrophysical scenarios, it could also occur in relativistic heavy-ion collisions, whenever the bombarding energy is large enough to form relatively cold, baryon-rich quark-gluon matter. The maximum amount of baryon stopping was found in heavy-ion collisions at AGS energies, $E_{\text {Lab }} \sim 10 \mathrm{AGeV}$; therefore, the most favorable conditions to form a color-superconducting phase of quark-gluon matter would occur in nuclear collisions somewhere in the energy range from $E_{\mathrm{Lab}} \sim 1$ to $40 \mathrm{AGeV}$, i.e., from GSI-SIS to CERN-SPS energies.

Most studies of color superconductivity assumed that only the two lightest quark flavors, $u$ and $d$, form Cooper pairs. This is based on the argument that, due to the larger strange quark mass, $m_{s} \simeq 100 \mathrm{MeV}$, 
the Fermi surfaces of non-strange and strange quarks do not match [3], consequently there are no us or $d s$ Cooper pairs. However, for a system where $\mu_{s}=\mu_{u}=\mu_{d} \equiv \mu$, this mismatch is, to leading order in $\mu$, only of order $\left(k_{F, q}-k_{F, s}\right) / \mu \sim\left(m_{s}^{2}-m_{q}^{2}\right) / 2 \mu^{2}$, and therefore vanishes at sufficiently large chemical potential. Based on this observation, Alford, Rajagopal, and Wilczek suggested a new form of color superconductivity, where all three flavors condense to lock the breaking of color and flavor symmetry [9]. Comparison with earlier investigations [10] of the patterns of symmetry breaking in chiral models revealed [11] that the colorflavor locked state is energetically favored. This was independently confirmed by Schäfer and Wilczek in a numerical study of the effective potential 12 .

Other studies which investigated the interplay of color superconductivity and chiral symmetry breaking at finite temperature include those of Berges and Rajagopal [13] and Langfeld and Rho 14. Color superconductivity was also investigated via renormalization group techniques by Evans, Hsu, and Schwetz [15] and by Schäfer and Wilczek [16.

All of these studies were based exclusively on NJL-type models. The sole exception is the work of Son 17] who applied renormalization group techniques to study the scale of the energy gap in QCD. He pointed out that non-instantaneous one-gluon exchange modifies the weak-coupling expression for the gap, $|\phi| \sim$ $\mu \exp \left(-c / g^{2}\right)$ to $|\phi| \sim \mu \exp (-c / g)$; this was also noted in 11. This increases the likelihood to find color superconductivity at nonzero temperature.

A quark-quark condensate $\Delta_{f g, \alpha \beta}^{i j}$ is a $N_{c} \times N_{c}$ matrix in color space $\left(i, j=1, \ldots, N_{c}\right)$, a $N_{f} \times N_{f}$ matrix in flavor space $\left(f, g=1, \ldots, N_{f}\right)$, and a $4 \times 4$ matrix in Dirac space $(\alpha, \beta=1, \ldots, 4)$. Quark pair condensation can in principle occur in channels with arbitrary total spin $J$. Most previous studies indicate, however, that condensation in the channel with total spin $J=0$ is favored for two or more flavors.

In this work, we shall not discuss the color or flavor structure of the gap matrix [18], but rather focus on the Dirac structure. To do so, it is sufficient to study a simpler model, where fermions interact via the exchange of scalar bosons,

$$
\mathcal{L}=\bar{\psi}(i \gamma \cdot \partial-m) \psi-g \bar{\psi} \psi \phi+\frac{1}{2}\left(\partial_{\mu} \phi \partial^{\mu} \phi-M_{s}^{2} \phi^{2}\right) .
$$

(We do not add a quartic self-interaction for the scalar field; such an interaction will not qualitatively change our results.) Scalar one-boson exchange is attractive, therefore, we expect the formation of Cooper pairs at finite density and sufficiently low temperature in the $J=0$ channel. Since the fermions in the Lagrangian (11) are not charged, this model can only exhibit superfluidity, not superconductivity. Nevertheless, the Dirac structure of the superfluid condensate is closely analogous to that of the color-superconducting condensate in QCD.

Our principal result is the following. Massless fermions have four types of $J=0$ condensates, corresponding to the pairing of fermions with the same helicity and chirality [11]. For fermions interacting via Yukawa interactions as in (1), the gap for right-handed, positive helicity quasiparticles is equal in magnitude (and opposite in sign) to that for left-handed, negative helicity quasiparticles. A novel feature of the relativistic treatment is the appearance of gaps for quasi-antiparticles. With Yukawa interactions, we find that the gap for right-handed, negative helicity quasi-antiparticles is equal in magnitude to that for left-handed, positive helicity quasi-antiparticles. These relations between the right- and left-handed gaps imply that there is condensation only in the $J^{P}=0^{+}$channel. For $M_{s}<\infty$, the quasiparticle and quasi-antiparticle gaps are not equal.

In the limit $M_{s} \rightarrow \infty$, the model (11) reduces to an NJL-type model, and the quasiparticle and quasiantiparticle gaps become degenerate. Thus NJL-type models are unrealistic, in that they force the equality of the quasiparticle and quasi-antiparticle gaps.

The number of condensates is not merely a technical matter, but can even affect the order of a superconducting phase transition. Consider the transition where up and down quarks condense to a colorsuperconducting phase. In [11] we demonstrated that if only one condensate appears, such as that for particles, the transition is in the universality class of a single $U(3)$ vector and can be of second order. Based on the analysis of this paper, it seems unavoidable that condensates for both particles and antiparticles appear together. The universality class becomes that of two $U(3)$ vectors; while the fixed-point structure of this model is unknown, it might be driven first order by the Coleman-Weinberg phenomenon. If true, it implies that for two degenerate flavors, the color-superconducting transition is of first order, independent of the chiral transition [13].

In [11] we also showed that the transition where up and down quarks condense with strange quarks is driven first order by the Coleman-Weinberg mechanism. Schäfer and Wilczek argued that the degrees of 
freedom in quark and hadron matter match at nonzero $\mu[12$. Their observation implies that this line of first order transitions, which starts at zero temperature, could terminate at some nonzero temperature, similar to the liquid-gas transition in water or nuclear matter.

The outline of the paper is as follows. In Section II we compute the full fermion propagator and discuss the excitation spectrum in a superfluid. Then, the gap equations are derived in the mean-field approximation for the model (1). We solve them in Section III. A logarithmic ultraviolet singularity arises, which upon renormalization introduces a renormalization scale $\Lambda$. We study solutions of the gap equations as a function of the coupling $g$ and the renormalization scale $\Lambda$. We also analyze the temperature dependence of the gap energy and discuss the critical temperature $T_{c}$, at which superfluidity vanishes. Section IV concludes with a summary of our results. Appendix A contains a derivation of the grand partition function and the gap equation in the mean-field approximation, both for an antifermion-fermion condensate and a fermion-fermion condensate. In Appendix B we analyze the Dirac structure of the gap matrix in detail and make contact to earlier work [3]. In Appendix $\mathrm{C}$ we discuss the renormalization of the gap equations. Our units are $\hbar=k_{B}=c=1$. The metric tensor is $g^{\mu \nu}=(+,-,-,-)$.

\section{THE GAP EQUATIONS IN THE ULTRARELATIVISTIC LIMIT}

A standard way to study superfluidity is to compute the grand partition function $\mathcal{Z}(T, V, \mu)$ associated with (11) in the mean-field approximation for a fermion-fermion condensate. This is done explicitly in Appendix A], cf. eq. (A15). One arrives at an effective action of the form (cf. eq. (A16), see also eq. (1.1) of [3])

$$
I[\bar{\psi}, \psi]=\int_{x} \bar{\psi}(x)\left(i \gamma \cdot \partial+\mu \gamma_{0}-m\right) \psi(x)+\frac{1}{2} \int_{x, y}\left[\bar{\psi}_{C}(x) \Delta(x, y) \psi(y)+\text { h.c. }\right]
$$

where $\psi_{C}(x)$ is the charge-conjugate spinor, defined by

$$
\psi_{C}(x)=C \bar{\psi}^{T}(x), \bar{\psi}_{C}(x)=\psi^{T}(x) C, \quad \psi(x)=C \bar{\psi}_{C}^{T}(x), \quad \bar{\psi}(x)=\psi_{C}^{T}(x) C
$$

and $C=i \gamma^{2} \gamma_{0}$ is the charge-conjugation matrix in Dirac representation, $C=-C^{-1}=-C^{T}=-C^{\dagger}$. We also abbreviated $\int_{x} \equiv \int_{0}^{1 / T} \mathrm{~d}(i t) \int_{V} \mathrm{~d}^{3} \mathbf{x} . \Delta(x, y)$ is the gap matrix. If the system is translationally invariant, $\Delta(x, y) \equiv \Delta(x-y)$, the Fourier transform

$$
\Delta(k)=\int_{x} e^{i k \cdot x} \Delta(x)
$$

obeys the gap equation (cf. eq. (A35), see also eq. (1.34) of [3])

$$
\Delta(k)=g^{2} \frac{T}{V} \sum_{q} D\left(k-q, M_{s}\right) G_{0}^{-}(q) \Delta(q) G^{+}(q) .
$$

Here, $k_{0}=-i(2 n+1) \pi T, \sum_{k} \equiv \sum_{n} V \int \mathrm{d}^{3} \mathbf{k} /(2 \pi)^{3}$, and

$$
\left[G_{0}^{ \pm}\right]^{-1}(k) \equiv \gamma \cdot k \pm \mu \gamma_{0}-m
$$

is the free inverse fermion propagator, while

$$
G^{ \pm} \equiv\left\{\left[G_{0}^{ \pm}\right]^{-1}-\Sigma^{ \pm}\right\}^{-1}
$$

is the fermion propagator dressed by the interaction with the fermion-fermion condensate, $\Sigma^{ \pm} \equiv$ $\Delta^{\mp} G_{0}^{\mp} \Delta^{ \pm}, \Delta^{+} \equiv \Delta, \Delta^{-} \equiv \gamma_{0} \Delta^{\dagger} \gamma_{0} . G_{0}^{+}$corresponds to propagation of free particles, $G_{0}^{-}$to that of charge-conjugate particles. $G^{+}$propagates quasiparticles and $G^{-}$charge-conjugate quasiparticles, respectively. $D\left(p, M_{s}\right)=1 /\left(M_{s}^{2}-p^{2}\right)$ is the propagator of the scalar boson.

In Appendix B we show that massive fermions have eight possible gaps in the $J=0$ channel. In the ultrarelativistic limit, these reduce to four. They correspond to the pairing of right-handed fields with positive helicity with themselves, etc. Consequently, the gap matrix (4) has the form 


$$
\Delta(k)=\phi_{r+}^{+}(k) \mathcal{P}_{r+}^{+}(\mathbf{k})+\phi_{\ell-}^{+}(k) \mathcal{P}_{\ell-}^{+}(\mathbf{k})+\phi_{r-}^{-}(k) \mathcal{P}_{r-}^{-}(\mathbf{k})+\phi_{\ell+}^{-}(k) \mathcal{P}_{\ell+}^{-}(\mathbf{k})
$$

Here,

$$
\mathcal{P}_{r+}^{+}(\mathbf{k}) \equiv \mathcal{P}_{r} \mathcal{P}_{+}(\mathbf{k}) \quad, \quad \mathcal{P}_{\ell-}^{+}(\mathbf{k}) \equiv \mathcal{P}_{\ell} \mathcal{P}_{-}(\mathbf{k}) \quad, \quad \mathcal{P}_{r-}^{-}(\mathbf{k}) \equiv \mathcal{P}_{r} \mathcal{P}_{-}(\mathbf{k}), \quad \mathcal{P}_{\ell+}^{-}(\mathbf{k}) \equiv \mathcal{P}_{\ell} \mathcal{P}_{+}(\mathbf{k})
$$

are projectors onto states with given chirality and helicity, as denoted by the two subscripts, where

$$
\mathcal{P}_{r, \ell} \equiv \frac{1 \pm \gamma_{5}}{2}, \quad \mathcal{P}_{ \pm}(\mathbf{k}) \equiv \frac{1 \pm \gamma_{5} \gamma_{0} \gamma \cdot \hat{\mathbf{k}}}{2}
$$

The additional superscript in (9) refers to the sign of the energy for a non-interacting fermion field. For massless fermions, this superscript is in principle superfluous: right- (left-) handed fermions with positive (negative) helicity must have positive energy, while those with negative (positive) helicity must have negative energy. Nevertheless, we find it physically illuminating to retain it.

The quantities $\phi_{r, \ell \pm}^{ \pm}$are the individual gap functions; for example, $\phi_{r+}^{+}$corresponds to the condensation of two right-handed particles with positive helicity, while $\phi_{r_{-}}^{-}$corresponds to the condensation of two righthanded antiparticles with negative helicity. This means that in the $J=0$ channel, fermions of different helicity or chirality do not condense. This is due to the projectors in eq. (8): the effective action (2) reduces to a sum of four terms, one for each given chirality and helicity, see eq. (B34).

The fact that only fermions with the same helicity condense in the $J=0$ channel is physically obvious in the center-of-momentum frame of the pair, and holds for either massive or massless fermions. In that frame, the requirement of equal helicity means that the projections of the spin along the direction of motion are antiparallel, as in the usual non-relativistic treatment of superconductivity. Taking the fermions to move in the $z$ direction, in the center-of-momentum frame $L_{z}=0$; thus if $J=0$, also $S_{z}=0$. The spin wave function with $S_{z}=0$ can be either the antisymmetric singlet, $S=0$, or the symmetric triplet, $S=1$. To obtain total spin $J=0$, for the former, $S=0$ combines with $L=0$, while for the latter, $S=1$ combines with $L=1$. The signal for $L=1$ is the appearance of $\hat{\mathbf{k}}$ in eqs. (8), (10).

In the following, we first discuss the structure of the full fermion propagator ( full propagator determine the excitation spectrum in a superfluid. We then return to the gap equation (5).

\section{A. The full fermion propagator}

In the ultrarelativistic limit, the full propagator (7) assumes the form

$$
G^{+}(q)=\left[\gamma \cdot q+\mu \gamma_{0}-\gamma_{0}[\Delta(q)]^{\dagger} \gamma_{0}\left(\gamma \cdot q-\mu \gamma_{0}\right)^{-1} \Delta(q)\right]^{-1}
$$

From eq. (8) one computes

$$
\gamma_{0} \Delta^{\dagger} \gamma_{0}=\left[\phi_{r+}^{+}\right]^{\dagger} \mathcal{P}_{\ell+}^{-}+\left[\phi_{\ell-}^{+}\right]^{\dagger} \mathcal{P}_{r-}^{-}+\left[\phi_{r-}^{-}\right]^{\dagger} \mathcal{P}_{\ell-}^{+}+\left[\phi_{\ell+}^{-}\right]^{\dagger} \mathcal{P}_{r+}^{+} .
$$

Since the condensates $\phi$ are not matrices (i.e., unlike the QCD case, they do not carry other internal degrees of freedom like color and flavor), the hermitean conjugation is replaced by simple complex conjugation. With the identities

$$
\gamma_{0} \Delta^{\dagger} \gamma_{0}\left(\gamma \cdot q-\mu \gamma_{0}\right) \Delta=\left(\gamma \cdot q-\mu \gamma_{0}\right)\left(\left|\phi_{r+}^{+}\right|^{2} \mathcal{P}_{r+}^{+}+\left|\phi_{\ell-}^{+}\right|^{2} \mathcal{P}_{\ell-}^{+}+\left|\phi_{r-}^{-}\right|^{2} \mathcal{P}_{r-}^{-}+\left|\phi_{\ell+}^{-}\right|^{2} \mathcal{P}_{\ell+}^{-}\right)
$$

and

$$
\left(\gamma \cdot q-\mu \gamma_{0}\right)\left(\gamma \cdot q+\mu \gamma_{0}\right)=\left[q_{0}^{2}-(|\mathbf{q}|-\mu)^{2}\right]\left(\mathcal{P}_{r+}^{+}+\mathcal{P}_{\ell-}^{+}\right)+\left[q_{0}^{2}-(|\mathbf{q}|+\mu)^{2}\right]\left(\mathcal{P}_{r-}^{-}+\mathcal{P}_{\ell+}^{-}\right),
$$

one derives

$$
G^{+}(q)=\left[\frac{\mathcal{P}_{r+}^{+}(\mathbf{q})}{q_{0}^{2}-\left[\epsilon^{+}\left(\phi_{r+}^{+}\right)\right]^{2}}+\frac{\mathcal{P}_{\ell-}^{+}(\mathbf{q})}{q_{0}^{2}-\left[\epsilon^{+}\left(\phi_{\ell-}^{+}\right)\right]^{2}}+\frac{\mathcal{P}_{r-}^{-}(\mathbf{q})}{q_{0}^{2}-\left[\epsilon^{-}\left(\phi_{r-}^{-}\right)\right]^{2}}+\frac{\mathcal{P}_{\ell+}^{-}(\mathbf{q})}{q_{0}^{2}-\left[\epsilon^{-}\left(\phi_{\ell+}^{-}\right)\right]^{2}}\right]\left(\gamma \cdot q-\mu \gamma_{0}\right),
$$

where 


$$
\epsilon^{ \pm}(\phi) \equiv\left[(|\mathbf{q}| \mp \mu)^{2}+|\phi|^{2}\right]^{1 / 2}
$$

The full propagator (15) has eight poles in the complex $q_{0}$ plane:

$$
q_{0}^{(1,2)}= \pm \epsilon^{+}\left(\phi_{r+}^{+}\right) \quad, \quad q_{0}^{(3,4)}= \pm \epsilon^{+}\left(\phi_{\ell-}^{+}\right) \quad, \quad q_{0}^{(5,6)}= \pm \epsilon^{-}\left(\phi_{r-}^{-}\right), \quad q_{0}^{(7,8)}= \pm \epsilon^{-}\left(\phi_{\ell+}^{-}\right) .
$$

These poles deserve further discussion. The kinetic energy of non-interacting, massless particles with 3momentum $\mathbf{q}$ is $|\mathbf{q}|$. Let $\omega(|\mathbf{q}|)$ denote the energy of the quasiparticle excitations as a function of the kinetic energy of the non-interacting particles. In a non-interacting, ultrarelativistic Fermi system quasiparticles (quasi-antiparticles) are the usual particles (antiparticles) and consequently the excitation spectrum is

$$
\begin{aligned}
\omega_{0}(|\mathbf{q}|)-\mu & =+|\mathbf{q}|-\mu & & \text { particles }, \\
& =-|\mathbf{q}|+\mu & & \text { particle holes }, \\
& =-|\mathbf{q}|-\mu & & \text { antiparticles }, \\
& =+|\mathbf{q}|+\mu & & \text { antiparticle holes } .
\end{aligned}
$$

On the other hand, in a superfluid the pairing of particles and antiparticles changes the excitation spectrum. As is well-known [1], the excitations are now quasiparticles (quasi-antiparticles) which are in essence a linear superposition of particles (antiparticles) and particle holes (antiparticle holes). This linear superposition is the well-known Bogoliubov transformation [1]. The quasiparticle excitations are sometimes called "Bogoliubons". On account of (17), in a superfluid the spectrum of right-handed excitations is

$$
\begin{aligned}
\omega(|\mathbf{q}|)-\mu & \equiv-\epsilon^{+}\left(\phi_{r+}^{+}\right)=-\sqrt{(|\mathbf{q}|-\mu)^{2}+\left|\phi_{r+}^{+}\right|^{2}} \\
& \equiv+\epsilon^{+}\left(\phi_{r+}^{+}\right)=+\sqrt{(|\mathbf{q}|-\mu)^{2}+\left|\phi_{r+}^{+}\right|^{2}} \\
& \equiv-\epsilon^{-}\left(\phi_{r-}^{-}\right)=-\sqrt{(|\mathbf{q}|+\mu)^{2}+\left|\phi_{r-}^{-}\right|^{2}} \\
& \equiv+\epsilon^{-}\left(\phi_{r-}^{-}\right)=+\sqrt{(|\mathbf{q}|+\mu)^{2}+\left|\phi_{r-}^{-}\right|^{2}}
\end{aligned}
$$

right-handed quasiparticles , right-handed quasiparticle holes , right-handed quasi-antiparticles , right-handed quasi-antiparticle holes ,

while for left-handed excitations one has

$$
\begin{aligned}
\omega(|\mathbf{q}|)-\mu & \equiv-\epsilon^{+}\left(\phi_{\ell-}^{+}\right)=-\sqrt{(|\mathbf{q}|-\mu)^{2}+\left|\phi_{\ell-}^{+}\right|^{2}} & & \text { left-handed quasiparticles } \\
& \equiv+\epsilon^{+}\left(\phi_{\ell-}^{+}\right)=+\sqrt{(|\mathbf{q}|-\mu)^{2}+\left|\phi_{\ell-}^{+}\right|^{2}} & & \text { left-handed quasiparticle holes } \\
& \equiv-\epsilon^{-}\left(\phi_{\ell+}^{-}\right)=-\sqrt{(|\mathbf{q}|+\mu)^{2}+\left|\phi_{\ell+}^{-}\right|^{2}} & & \text { left-handed quasi-antiparticles } \\
& \equiv+\epsilon^{-}\left(\phi_{\ell+}^{-}\right)=+\sqrt{(|\mathbf{q}|+\mu)^{2}+\left|\phi_{\ell+}^{-}\right|^{2}} & & \text { left-handed quasi-antiparticle holes }
\end{aligned}
$$

These branches are displayed in Fig. 1, for simplicity with a common gap $\phi=0.5 \mu$. For free fermions, the particle and hole branches cross at the Fermi surface. The generation of a gap produces a phenomenon analogous to level repulsion in quantum mechanics, as the quasiparticle and quasiparticle-hole branches become disconnected. Relative to the non-interacting case, the meaning of quasiparticle and quasiparticlehole branch interchanges above the Fermi surface.

The main difference from the non-relativistic case is the appearance of the (quasi-)antiparticle branch and the (quasi-)antiparticle-hole branch. This means that condensation is not only restricted to particles close to the Fermi surface, but that also antiparticles in the Dirac sea condense. This can be confirmed by considering the fermion-fermion scattering amplitude: a BCS-type singularity occurs not only for particleparticle scattering, but also for antiparticle-antiparticle scattering, giving rise to the antiparticle condensates $\phi_{r-, \ell+}^{-}$. Notice, however, that it is always easier to excite a quasiparticle than a quasi-antiparticle. It costs only an energy $2 \phi$ to excite a quasiparticle at the Fermi surface, but $2 \sqrt{\mu^{2}+\phi^{2}}$ to excite a quasi-antiparticle with zero momentum. 


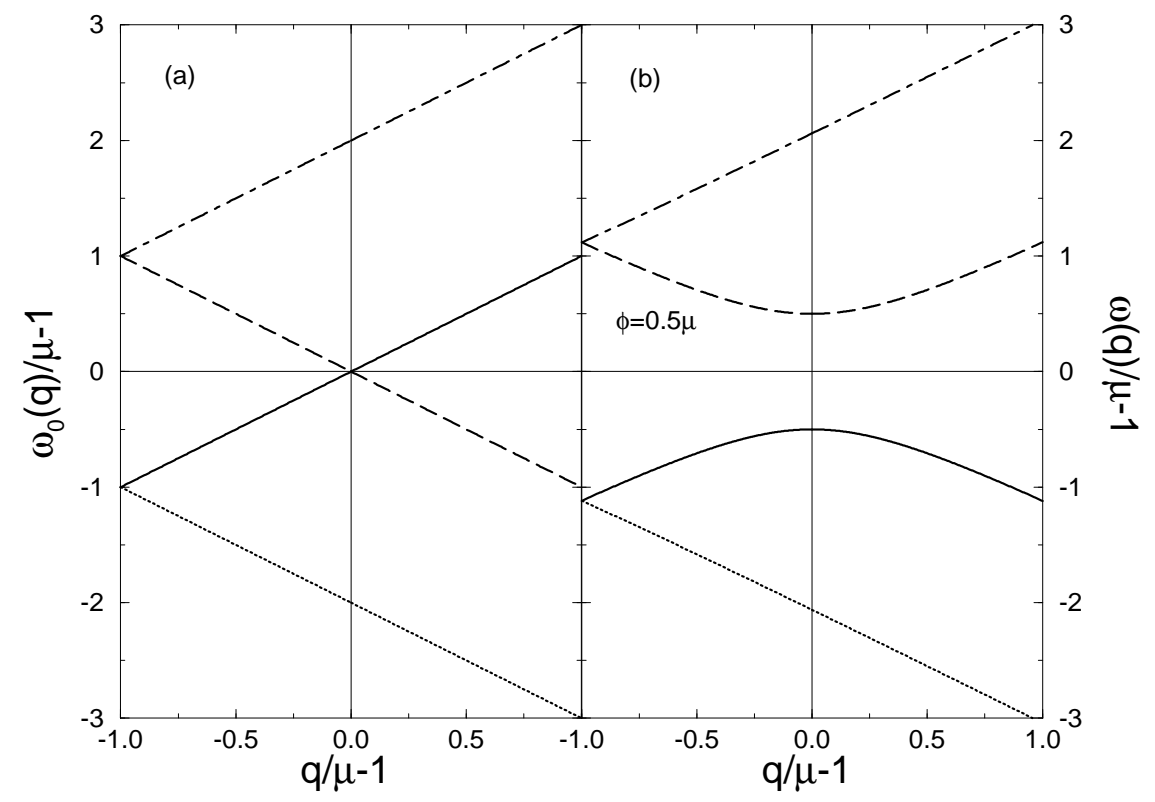

FIG. 1. The dispersion relation for (quasi)particle (solid), (quasi-)antiparticle (dotted), (quasi)particle-hole (dashed), and (quasi-)antiparticle-hole states (dot-dashed). (a) Non-interacting, massless fermions, (b) superfluid, massless fermions.

One can also compute the occupation numbers for quasiparticles and quasi-antiparticles. To this end, it is advantageous to Fourier-transform the full propagator to the so-called "mixed" (or "Saclay") representation

$$
G^{+}(\tau, \mathbf{q})=T \sum_{n} e^{i \omega_{n} \tau} G^{+}\left(\omega_{n}, \mathbf{q}\right)
$$

where $i \omega_{n}=i(2 n+1) \pi T \equiv-q_{0}$. For instance, the free propagator for massless fermions reads in this representation 19

$$
\begin{aligned}
G_{0}^{+}(\tau, \mathbf{q})= & -\left[\theta(\tau)-N_{F}\left(\frac{|\mathbf{q}|-\mu}{T}\right)\right] \exp [-(|\mathbf{q}|-\mu) \tau] \Lambda^{+}(\mathbf{q}) \gamma_{0} \\
& +\left[\theta(-\tau)-N_{F}\left(\frac{|\mathbf{q}|+\mu}{T}\right)\right] \exp [(|\mathbf{q}|+\mu) \tau] \Lambda^{-}(\mathbf{q}) \gamma_{0},
\end{aligned}
$$

where $N_{F}(x) \equiv\left(e^{x}+1\right)^{-1}$ is the usual Fermi-Dirac distribution and $\Lambda^{ \pm}(\mathbf{q})$ is the projector onto positive (negative) energy states (eq. (B22) in the massless limit, i.e., for $\beta_{\mathbf{q}}=1$ and $\alpha_{\mathbf{q}}=0$ ). The occupation numbers are now directly exhibited as the prefactors of the exponential (imaginary) time evolution factors. For $\tau \geq 0$, the first term corresponds to the propagation of particle holes, with the thermal occupation number $1-N_{F}[(|\mathbf{q}|-\mu) / T]$, while the second term corresponds to the propagation of antiparticles, with the thermal occupation number $N_{F}[(|\mathbf{q}|+\mu) / T]$. For $\tau<0$, the first term corresponds to the propagation of particles, with the thermal occupation number $N_{F}[(|\mathbf{q}|-\mu) / T]$. The second corresponds to the propagation of antiparticle holes, with occupation number $1-N_{F}[(|\mathbf{q}|+\mu) / T]$.

The full fermion propagator is computed from eq. (21) with (15). The result is

$$
\begin{aligned}
G^{+}(\tau, \mathbf{q})= & -\sum_{h s=r+, \ell-}\left(\left\{\theta(\tau)-N_{F}\left[\frac{\epsilon^{+}\left(\phi_{h s}^{+}\right)}{T}\right]\right\}\left[1-n_{\mathbf{q}}^{+}\left(\phi_{h s}^{+}\right)\right] \exp \left[-\epsilon^{+}\left(\phi_{h s}^{+}\right) \tau\right]\right. \\
& \left.-\left\{\theta(-\tau)-N_{F}\left[\frac{\epsilon^{+}\left(\phi_{h s}^{+}\right)}{T}\right]\right\} n_{\mathbf{q}}^{+}\left(\phi_{h s}^{+}\right) \exp \left[\epsilon^{+}\left(\phi_{h s}^{+}\right) \tau\right]\right) \mathcal{P}_{h s}^{+}(\mathbf{q}) \gamma_{0} \\
& -\sum_{h s=r-, \ell+}\left(\left\{\theta(\tau)-N_{F}\left[\frac{\epsilon^{-}\left(\phi_{h s}^{-}\right)}{T}\right]\right\}\left[1-n_{\mathbf{q}}^{-}\left(\phi_{h s}^{-}\right)\right] \exp \left[-\epsilon^{-}\left(\phi_{h s}^{-}\right) \tau\right]\right.
\end{aligned}
$$




$$
\left.-\left\{\theta(-\tau)-N_{F}\left[\frac{\epsilon^{-}\left(\phi_{h s}^{-}\right)}{T}\right]\right\} n_{\mathbf{q}}^{-}\left(\phi_{h s}^{-}\right) \exp \left[\epsilon^{-}\left(\phi_{h s}^{-}\right) \tau\right]\right) \mathcal{P}_{h s}^{-}(\mathbf{q}) \gamma_{0}
$$

where

$$
n_{\mathbf{q}}^{ \pm}(\phi) \equiv \frac{\epsilon^{ \pm}(\phi) \mp(|\mathbf{q}| \mp \mu)}{2 \epsilon^{ \pm}(\phi)}
$$

In deriving eq. (23), in an intermediate step one encounters terms of the form

$$
\frac{\left(\epsilon^{ \pm}-\mu\right) \gamma_{0}-\gamma \cdot \mathbf{q}}{2 \epsilon^{ \pm}} \quad \text { and } \quad \frac{-\left(\epsilon^{ \pm}+\mu\right) \gamma_{0}-\gamma \cdot \mathbf{q}}{2 \epsilon^{ \pm}} .
$$

By adding and subtracting a term $|\mathbf{q}| \gamma_{0}$ in the numerator, one can transform them into

$$
\gamma_{0}\left(1-n_{\mathbf{q}}^{ \pm}\right) \mp \Lambda^{\mp}(\mathbf{q}) \gamma_{0} \frac{|\mathbf{q}|}{\epsilon^{ \pm}} \quad \text { and } \quad-\gamma_{0} n_{\mathbf{q}}^{ \pm} \mp \Lambda^{\mp}(\mathbf{q}) \gamma_{0} \frac{|\mathbf{q}|}{\epsilon^{ \pm}}
$$

Eq. (23) then follows by making use of eq. (B31) and the orthogonality of the energy projectors, $\Lambda^{+} \Lambda^{-} \equiv 0$.

There is a one-to-one correspondence between the eight poles of the full propagator (17) and the eight terms on the right-hand side of eq. (23). The first line in eq. (23) arises from the pole for right(left)handed quasiparticle holes, the second line from that for right(left)-handed quasiparticles, while the third line originates from the pole for right(left)-handed quasi-antiparticle holes and the fourth line from that for right(left)-handed quasi-antiparticles. As in the non-interacting case (22), the occupation numbers can now be directly read off as the prefactors of the exponential time evolution factors.

It is interesting to study two limiting cases of (23). The first is the zero-temperature limit, where the FermiDirac distributions vanish on account of the fact that their argument is always positive. Then, the functions $n_{\mathbf{q}}^{ \pm}$defined in eq. (24) are identified with the occupation numbers for quasiparticles and quasi-antiparticles in a superfluid at $T=0$. Correspondingly, $1-n_{\mathbf{q}}^{ \pm}$are the occupation numbers of quasiparticle holes and quasi-antiparticle holes, respectively. These are shown in Fig. 2. The quasiparticle and quasiparticlehole occupation numbers exhibit the smearing around the Fermi surface characteristic for a superfluid or a superconductor [1]. The smearing is a consequence of the fact that the "Bogoliubons" are superpositions of particle and hole states. Note also that there are always some quasi-antiparticle-hole excitations present.

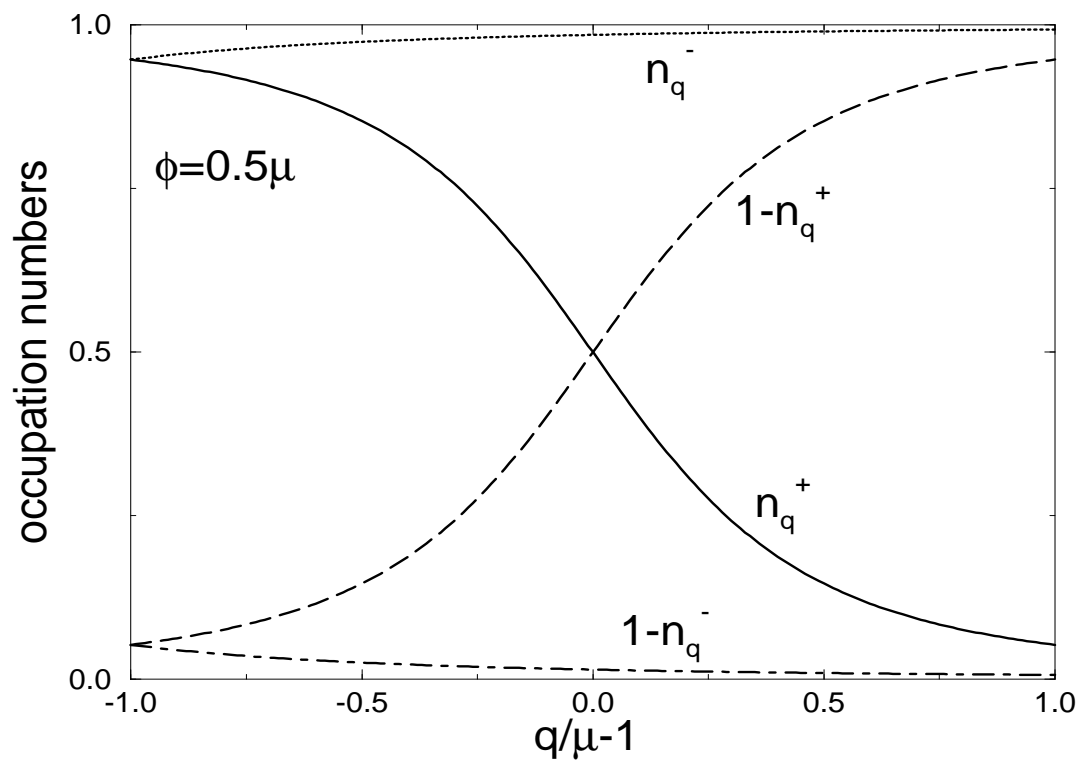

FIG. 2. The occupation number for quasiparticles $n_{\mathbf{q}}^{+}$(solid), quasi-antiparticles $n_{\mathbf{q}}^{-}$(dotted), quasiparticle holes $1-n_{\mathbf{q}}^{+}$(dashed), and quasi-antiparticle holes $1-n_{\mathbf{q}}^{-}$(dot-dashed) in a superfluid at $T=0$. 
The other interesting limit is the non-interacting case where one is supposed to recover eq. (22). Taking all gaps to vanish, $\phi_{h s}^{ \pm} \rightarrow 0$, one has $\epsilon^{ \pm} \rightarrow|| \mathbf{q}|\mp \mu|$, such that

$$
n_{\mathbf{q}}^{+} \rightarrow \theta(\mu-|\mathbf{q}|) \quad, \quad n_{\mathbf{q}}^{-} \rightarrow 1 \quad(\phi \rightarrow 0) .
$$

The contribution from quasi-antiparticle holes vanishes completely, and the quasi-antiparticle term becomes the antiparticle term, the second line of eq. (22). However, in order to obtain the particle-hole contribution (the first line of eq. (22)), one has to combine the contribution from quasiparticles and quasiparticle holes. This can be understood by inverting the Bogoliubov transformation which combines particles and particle holes to quasiparticles (and quasiparticle holes).

\section{B. The gap equations}

After discussing the full fermion propagator and the excitation spectrum in a superfluid, we now return to the discussion of the gap equation (5) in the ultrarelativistic limit. With the expression (15) for the full propagator, it assumes the form

$$
\begin{aligned}
& \Delta(k)=g^{2} \frac{T}{V} \sum_{q} D\left(k-q, M_{s}\right) {\left[\frac{\phi_{r+}^{+}(q)}{q_{0}^{2}-\left[\epsilon^{+}\left(\phi_{r+}^{+}\right)\right]^{2}} \mathcal{P}_{\ell+}^{-}(\mathbf{q})+\frac{\phi_{\ell-}^{+}(q)}{q_{0}^{2}-\left[\epsilon^{+}\left(\phi_{\ell-}^{+}\right)\right]^{2}} \mathcal{P}_{r-}^{-}(\mathbf{q})\right.} \\
&\left.+\frac{\phi_{r-}^{-}(q)}{q_{0}^{2}-\left[\epsilon^{-}\left(\phi_{r-}^{-}\right)\right]^{2}} \mathcal{P}_{\ell-}^{+}(\mathbf{q})+\frac{\phi_{\ell+}^{-}(q)}{q_{0}^{2}-\left[\epsilon^{-}\left(\phi_{\ell+}^{-}\right)\right]^{2}} \mathcal{P}_{r+}^{+}(\mathbf{q})\right] .
\end{aligned}
$$

Forming suitable projections of eq. (8), one derives gap equations for the individual gap functions:

$$
\begin{aligned}
& \phi_{r+}^{+}(k)=\frac{g^{2}}{2} \frac{T}{V} \sum_{q} D\left(k-q, M_{s}\right)\left[\frac{1-\hat{\mathbf{k}} \cdot \hat{\mathbf{q}}}{q_{0}^{2}-\left[\epsilon^{+}\left(\phi_{\ell-}^{+}\right)\right]^{2}} \phi_{\ell-}^{+}(q)+\frac{1+\hat{\mathbf{k}} \cdot \hat{\mathbf{q}}}{q_{0}^{2}-\left[\epsilon^{-}\left(\phi_{\ell+}^{-}\right)\right]^{2}} \phi_{\ell+}^{-}(q)\right], \\
& \phi_{\ell-}^{+}(k)=\frac{g^{2}}{2} \frac{T}{V} \sum_{q} D\left(k-q, M_{s}\right)\left[\frac{1-\hat{\mathbf{k}} \cdot \hat{\mathbf{q}}}{q_{0}^{2}-\left[\epsilon^{+}\left(\phi_{r+}^{+}\right)\right]^{2}} \phi_{r+}^{+}(q)+\frac{1+\hat{\mathbf{k}} \cdot \hat{\mathbf{q}}}{q_{0}^{2}-\left[\epsilon^{-}\left(\phi_{r-}^{-}\right)\right]^{2}} \phi_{r-}^{-}(q)\right], \\
& \phi_{r-}^{-}(k)=\frac{g^{2}}{2} \frac{T}{V} \sum_{q} D\left(k-q, M_{s}\right)\left[\frac{1+\hat{\mathbf{k}} \cdot \hat{\mathbf{q}}}{q_{0}^{2}-\left[\epsilon^{+}\left(\phi_{\ell-}^{+}\right)\right]^{2}} \phi_{\ell-}^{+}(q)+\frac{1-\hat{\mathbf{k}} \cdot \hat{\mathbf{q}}}{q_{0}^{2}-\left[\epsilon^{-}\left(\phi_{\ell+}^{-}\right)\right]^{2}} \phi_{\ell+}^{-}(q)\right], \\
& \phi_{\ell+}^{-}(k)=\frac{g^{2}}{2} \frac{T}{V} \sum_{q} D\left(k-q, M_{s}\right)\left[\frac{1+\hat{\mathbf{k}} \cdot \hat{\mathbf{q}}}{q_{0}^{2}-\left[\epsilon^{+}\left(\phi_{r+}^{+}\right)\right]^{2}} \phi_{r+}^{+}(q)+\frac{1-\hat{\mathbf{k}} \cdot \hat{\mathbf{q}}}{q_{0}^{2}-\left[\epsilon^{-}\left(\phi_{r-}^{-}\right)\right]^{2}} \phi_{r-}^{-}(q)\right] .
\end{aligned}
$$

These gap equations (27) do not agree with those derived by Bailin and Love [3]. These authors include only half of the fermion quasiparticle modes: they take the quasiparticle and quasiparticle-hole branches, with gaps $\phi_{r+}^{+}$and $\phi_{\ell-}^{+}$, but neglect the quasi-antiparticle and quasi-antiparticle-hole branches, with gaps $\phi_{r-}^{-}$and $\phi_{\ell_{+}}^{-}$. They also restrict themselves to condensation in the $0^{+}$channel, which forces $\phi_{r+}^{+}=-\phi_{\ell-}^{+}$ (see eq. (B35)). Doing so, they find a single equation for a linear combination of the gaps. We do not find a single equation. Instead, due to the form of the full fermion propagator (15), each of the four gap equations (27) contains a sum of two terms: each gap on the right-hand side, such as $\phi_{r+}^{+}$, is always multiplied by an energy denominator with only that gap, $1 /\left\{q_{0}^{2}-\left[\epsilon^{+}\left(\phi_{r+}^{+}\right)\right]^{2}\right\}$.

In the case of a point-like four-fermion interaction as in NJL-type models, $D\left(k-q, M_{s}\right) \rightarrow 1 / M_{s}^{2}$. Then, the terms proportional to $\hat{\mathbf{k}} \cdot \hat{\mathbf{q}}$ vanish by symmetry, and the right-hand sides of $(27)$ no longer depend on either $k_{0}$ or $\mathbf{k}$ : the gaps are simply constants. Defining

$$
F(\phi) \equiv \frac{g^{2}}{2 M_{s}^{2}} \frac{T}{V} \sum_{q}\left[\frac{1}{q_{0}^{2}-\left[\epsilon^{+}(\phi)\right]^{2}}+\frac{1}{q_{0}^{2}-\left[\epsilon^{-}(\phi)\right]^{2}}\right]
$$

the gap equations reduce to 


$$
\phi_{r+}^{+} \equiv \phi_{r-}^{-}=\phi_{\ell-}^{+} F\left(\phi_{\ell-}^{+}\right), \phi_{\ell-}^{+} \equiv \phi_{\ell+}^{-}=\phi_{r+}^{+} F\left(\phi_{r+}^{+}\right) .
$$

The gaps for right- and left-handed quasi-antiparticles equal those for the corresponding quasiparticles. This reduces the number of independent gaps to two.

The solutions of eqs. (29) satisfy $1=F(\phi) F(\phi F(\phi))$, where $\phi$ is either $\phi_{r+}^{+}$or $\phi_{\ell-}^{+}$. One possible solution, $\phi_{0}$, satisfies $1 \equiv\left|F\left(\phi_{0}\right)\right|$; as $F(\phi)$ is single-valued as a function of $\phi$ for $\phi \geq 0$, and $F(\phi)=F(-\phi)$, the only possible solutions are $\pm \phi_{0}$, therefore $\phi_{r+}^{+}= \pm \phi_{\ell_{-}}^{+}$. Since $F<0$, the solution of eqs. (29) obeys $\phi_{\ell-}^{+}=-\phi_{r+}^{+}$. From eq. (B35) we then conclude that condensation occurs only in the $0^{+}$channel.

In conclusion, for scalar NJL-type interactions there is only one independent gap function. Physically, this happens because for a point-like interaction there are no states with nonzero angular momentum, and so terms in the gap proportional to $\hat{\mathbf{k}}$ - which signal $L=1$ - must vanish. In the next section we show that scalar boson exchange over a finite range, $M_{s}<\infty$, lifts this degeneracy and produces two independent gap functions.

\section{SOLVING THE GAP EQUATIONS}

The set of equations (27) determines the gap functions for massless fermions in the mean-field approximation. In principle, the gaps are functions of the 4-momentum $k^{\mu}$, so that eqs. (27) are actually integral equations. In the following, we assume that the momentum dependence of the gap functions is negligible. We comment on the validity of this approximation below. Remember that in NJL-type models the gap functions are automatically independent of $k^{\mu}$.

Under the assumption that the gap functions are simply constants, one ends up with the following set of four algebraic equations:

$$
\begin{aligned}
& \phi_{r+}^{+}=\phi_{\ell-}^{+}\left[\mathcal{F}_{0}^{+}\left(\phi_{\ell-}^{+}\right)-\mathcal{F}_{1}^{+}\left(\phi_{\ell-}^{+}\right)\right]+\phi_{\ell+}^{-}\left[\mathcal{F}_{0}^{-}\left(\phi_{\ell+}^{-}\right)+\mathcal{F}_{1}^{-}\left(\phi_{\ell+}^{-}\right)\right], \\
& \phi_{\ell-}^{+}=\phi_{r+}^{+}\left[\mathcal{F}_{0}^{+}\left(\phi_{r+}^{+}\right)-\mathcal{F}_{1}^{+}\left(\phi_{r+}^{+}\right)\right]+\phi_{r-}^{-}\left[\mathcal{F}_{0}^{-}\left(\phi_{r-}^{-}\right)+\mathcal{F}_{1}^{-}\left(\phi_{r-}^{-}\right)\right], \\
& \phi_{r-}^{-}=\phi_{\ell-}^{+}\left[\mathcal{F}_{0}^{+}\left(\phi_{\ell-}^{+}\right)+\mathcal{F}_{1}^{+}\left(\phi_{\ell-}^{+}\right)\right]+\phi_{\ell+}^{-}\left[\mathcal{F}_{0}^{-}\left(\phi_{\ell+}^{-}\right)-\mathcal{F}_{1}^{-}\left(\phi_{\ell+}^{-}\right)\right], \\
& \phi_{\ell+}^{-}=\phi_{r+}^{+}\left[\mathcal{F}_{0}^{+}\left(\phi_{r+}^{+}\right)+\mathcal{F}_{1}^{+}\left(\phi_{r+}^{+}\right)\right]+\phi_{r-}^{-}\left[\mathcal{F}_{0}^{-}\left(\phi_{r-}^{-}\right)-\mathcal{F}_{1}^{-}\left(\phi_{r-}^{-}\right)\right],
\end{aligned}
$$

where

$$
\mathcal{F}_{n}^{ \pm}(\phi) \equiv \frac{g^{2}}{2} \frac{T}{V} \sum_{q} D\left(k-q, M_{s}\right) \frac{(\hat{\mathbf{k}} \cdot \hat{\mathbf{q}})^{n}}{q_{0}^{2}-\left[\epsilon^{ \pm}(\phi)\right]^{2}}
$$

Note that $\mathcal{F}_{1}^{ \pm} \equiv 0$ for NJL-type models.

The functions $\mathcal{F}_{0,1}^{ \pm}$are further evaluated replacing the Matsubara sum over $q_{0} \equiv-i(2 n+1) \pi T$ by a contour integral and applying the residue theorem. Poles in the complex $q_{0}$ plane arise from the fermion as well as the boson propagator. Keeping only the former, we obtain

$$
\begin{aligned}
& \mathcal{F}_{0}^{ \pm} \equiv-\frac{g^{2}}{32 \pi^{2} k} \int_{0}^{\infty} \mathrm{d} q \ln \left[\frac{M_{s}^{2}+(k+q)^{2}}{M_{s}^{2}+(k-q)^{2}}\right] \frac{q}{\epsilon^{ \pm}} \tanh \left[\frac{\epsilon^{ \pm}}{2 T}\right], \\
& \mathcal{F}_{1}^{ \pm} \equiv-\frac{g^{2}}{32 \pi^{2} k} \int_{0}^{\infty} \mathrm{d} q\left\{\frac{M_{s}^{2}+k^{2}+q^{2}}{2 q k} \ln \left[\frac{M_{s}^{2}+(k+q)^{2}}{M_{s}^{2}+(k-q)^{2}}\right]-2\right\} \frac{q}{\epsilon^{ \pm}} \tanh \left[\frac{\epsilon^{ \pm}}{2 T}\right],
\end{aligned}
$$

where we have performed the angular integration and denoted $k \equiv|\mathbf{k}|, q \equiv|\mathbf{q}|$. Since $\mathcal{F}_{0,1}^{ \pm}$are real, the gap functions $\phi$ can be chosen to be real.

To obtain this result we assume that the exchanged boson has zero energy, $q_{0}=k_{0}$. This approximation can be justified as follows. The dominant term in $\mathcal{F}_{0,1}^{+}$comes from particles close to the Fermi surface, $q \simeq \mu$. Assuming that $|\phi| \ll \mu$, this produces a logarithmic dependence on $|\phi|$ :

$$
\int_{0}^{\Lambda_{\mathrm{UV}}} \frac{\mathrm{d} q}{\epsilon^{+}}=\int_{0}^{\Lambda_{\mathrm{UV}}} \frac{\mathrm{d} q}{\sqrt{(q-\mu)^{2}+|\phi|^{2}}} \sim \ln \frac{\Lambda_{\mathrm{UV}}}{|\phi|} .
$$

(We discuss the ultraviolet cut-off $\Lambda_{\mathrm{UV}} \gg \mu$ below.) The assumption that the gap $|\phi|$ is small relative to the Fermi energy is justified in weak coupling, $g \ll 1$. To obtain the logarithm (33), the fermions have to stay close to the Fermi surface. This can only be achieved if the exchanged boson has zero energy, $q_{0}=k_{0}$. 
Poles of the boson propagator, $q_{0}=k_{0} \pm \sqrt{M_{s}^{2}+(\mathbf{k}-\mathbf{q})^{2}}$, which we neglected in deriving eqs. (32), represent processes in which the fermions in the gap equation are far from the Fermi surface; this produces terms of order 1 , but not of order $\ln \left(\Lambda_{\mathrm{UV}} /|\phi|\right)$. (Further, keeping only zero-energy bosons also eliminates a possible dependence of $\mathcal{F}_{0,1}^{ \pm}$on $k_{0}$.)

Analogous to eq. (33), the functions $\mathcal{F}_{0,1}^{-}$behave as

$$
\int_{0}^{\Lambda_{\mathrm{UV}}} \frac{\mathrm{d} q}{\epsilon^{-}}=\int_{0}^{\Lambda_{\mathrm{UV}}} \frac{\mathrm{d} q}{\sqrt{(q+\mu)^{2}+|\phi|^{2}}} \sim \ln \frac{\Lambda_{\mathrm{UV}}}{\mu}
$$

in the limit $\Lambda_{\mathrm{UV}} \gg \mu \gg|\phi|$. Therefore, $\mathcal{F}_{0,1}^{-}$do not include terms $\sim \ln \left(\Lambda_{\mathrm{UV}} /|\phi|\right)$ and, in weak coupling, can be neglected relative to $\mathcal{F}_{0,1}^{+}$. Physically, this is because $\epsilon^{-}$represents the excitation spectrum of quasiantiparticles which are always far from the Fermi surface, cf. Fig. 1.

While our approximations are controlled only in weak coupling, we nevertheless find it illustrative to consider the qualitative nature of the solutions in strong coupling. For $g \simeq 1$, the gap can be of order $\mu$. Similarly, the functions $\mathcal{F}_{0,1}^{-}$are of comparable magnitude to $\mathcal{F}_{0,1}^{+}$. We therefore retain them in the following analysis.

It is surprising that an ultraviolet cut-off $\Lambda_{\mathrm{UV}}$ appears in the gap equations. There is no ultraviolet divergence for the gap equations of ordinary superconductors [1], as a cut-off is provided by the Debye frequency, $\omega_{D} \ll \mu$, so that the integrals receive contributions only from a narrow interval around the Fermi surface, $\mu-\omega_{D} \leq q \leq \mu+\omega_{D}$. On the other hand, in $\mathrm{He}-3$ the cut-off is provided by the chemical potential, $\mu$.

The appearance of $\Lambda_{\mathrm{UV}}$ is an artefact of our approximation of taking a constant gap. This approximation is manifestly inconsistent when $k \gg \mu$. The gap functions fall off at large $k$, removing the apparent ultraviolet divergence. Consequently, the true gap function is proportional to $\mu$, not $\Lambda_{\mathrm{UV}}$. That there is no ultraviolet divergence in the true gap function can also be seen by considering the particle-particle and antiparticleantiparticle scattering amplitudes. The box diagrams whose infrared singularities generate a non-zero gap [1] are manifestly ultraviolet finite, even in the relativistic regime. In order to simplify the solution of the gap equations, however, we take the gap functions to be constant and thus ignore these complications.

With this approximation, the integral $\mathcal{F}_{0}^{ \pm}$is logarithmically divergent in the ultraviolet, while $\mathcal{F}_{1}^{ \pm}$is finite. Instead of using a fixed ultraviolet cut-off $\Lambda_{\mathrm{UV}}$, in Appendix $\mathbb{Q}$ we show how $\mathcal{F}_{0}^{ \pm}$can be rendered finite by renormalization. The result is

$$
\mathcal{F}_{0}^{ \pm}=-\frac{g^{2}}{32 \pi^{2} k} \int_{0}^{\infty} \mathrm{d} q\left\{\frac{q}{\epsilon^{ \pm}} \ln \left[\frac{M_{s}^{2}+(k+q)^{2}}{M_{s}^{2}+(k-q)^{2}}\right] \tanh \left[\frac{\epsilon^{ \pm}}{2 T}\right]-\ln \frac{(k+q)^{2}}{(k-q)^{2}}\right\}+\frac{g^{2}}{16 \pi^{2}} \ln \frac{k^{2}}{\Lambda^{2} e^{2}},
$$

where $\Lambda$ is a renormalization scale. One can readily convince oneself that for $\Lambda \gg k$, the renormalization scale can be identified with the ultraviolet cut-off $\Lambda_{\mathrm{UV}}$ introduced above. In light of this, we take $\Lambda \gg \mu$.

In the following, we turn to the solution of the gap equations, first at $T=0$ and then at nonzero $T$. It is instructive to start with the weak-coupling limit, $g \ll 1$.

\section{A. Weak-coupling limit}

The gap energy $\phi$ is expected to be exponentially small in weak coupling, $\phi \sim \mu \exp \left(-c / g^{2}\right)$ [1]. The integrands of the functions $\mathcal{F}_{0,1}^{+}$are strongly peaked around $q=\mu$, since then $\epsilon^{+}=|\phi| \ll \mu$. Consequently, the main contribution to the integrals $\mathcal{F}_{0,1}^{+}$comes from a (small) region $\mu-\delta \leq q \leq \mu+\delta$, where $\delta=a \mu$, with some constant $a$ which we do not determine. The functions $\mathcal{F}_{0,1}^{-}$are relatively suppressed by a factor $\epsilon^{+} / \epsilon^{-} \simeq|\phi| / 2 \mu \ll 1$. For $\delta \gg|\phi|$ :

$$
\begin{aligned}
& \mathcal{F}_{0}^{+} \simeq-\frac{g^{2}}{16 \pi^{2}} \frac{\mu}{k} \ln \left[\frac{M_{s}^{2}+(k+\mu)^{2}}{M_{s}^{2}+(k-\mu)^{2}}\right] \ln \frac{2 \delta}{|\phi|}, \\
& \mathcal{F}_{1}^{+} \simeq-\frac{g^{2}}{16 \pi^{2}} \frac{\mu}{k}\left\{\frac{M_{s}^{2}+k^{2}+\mu^{2}}{2 \mu k} \ln \left[\frac{M_{s}^{2}+(k+\mu)^{2}}{M_{s}^{2}+(k-\mu)^{2}}\right]-2\right\} \ln \frac{2 \delta}{|\phi|}, \\
& \mathcal{F}_{0}^{-} \simeq 0, \mathcal{F}_{1}^{-} \simeq 0 .
\end{aligned}
$$

Renormalization corrections are unimportant in this limit, since they only change $\delta$. 
The gap equations (30) were derived under the approximation that the gaps are constants independent of $k$. From eq. (36), however, we see that the functions $\mathcal{F}_{0,1}^{+}$do depend strongly on $k$, and peak around $k=\mu$. Thus, as was seen previously in eq. (33), in weak coupling pairing is dominated by fermions close to the Fermi surface. For $g \ll 1$ it is therefore consistent to neglect the momentum dependence of both $\mathcal{F}_{0,1}^{+}$and the gap functions and consider the above expressions (36) at $k=\mu$.

To one-loop order, a scalar mass is generated by its coupling to a fermion loop,

$$
M_{s}^{2}=\frac{g^{2}}{2}\left(\frac{\mu^{2}}{\pi^{2}}+T^{2}\right) .
$$

If we had included scalar quartic interactions in the Lagrangian (1), they would contribute to $M_{s}^{2}$ a term $\sim \lambda T^{2}$, where $\lambda$ is the quartic coupling. Thus, at zero temperature there is no change, while at nonzero temperature there is an innocuous increase of $M_{s}^{2}$. As will be disussed below, this tends to decrease $T_{c}$.

In weak coupling, $M_{s} \sim g \mu \ll \mu$, and we can further approximate

$$
\begin{aligned}
& \mathcal{F}_{0}^{+} \simeq-\frac{g^{2}}{16 \pi^{2}} \ln \frac{4 \mu^{2}}{M_{s}^{2}} \ln \frac{2 \delta}{|\phi|} \\
& \mathcal{F}_{1}^{+} \simeq-\frac{g^{2}}{16 \pi^{2}}\left[\ln \frac{4 \mu^{2}}{M_{s}^{2}}-2\right] \ln \frac{2 \delta}{|\phi|} .
\end{aligned}
$$

We can now solve the gap equations (30). Using (38) we obtain:

$$
\begin{aligned}
& \phi_{r+}^{+}=-\phi_{\ell-}^{+} \frac{g^{2}}{8 \pi^{2}} \ln \frac{2 \delta}{\left|\phi_{\ell-}^{+}\right|}, \\
& \phi_{\ell-}^{+}=-\phi_{r+}^{+} \frac{g^{2}}{8 \pi^{2}} \ln \frac{2 \delta}{\left|\phi_{r+}^{+}\right|}, \\
& \phi_{r-}^{-}=-\phi_{\ell-}^{+} \frac{g^{2}}{8 \pi^{2}}\left[\ln \frac{4 \mu^{2}}{M_{s}^{2}}-1\right] \ln \frac{2 \delta}{\left|\phi_{\ell-}^{+}\right|}, \\
& \phi_{\ell+}^{-}=-\phi_{r+}^{+} \frac{g^{2}}{8 \pi^{2}}\left[\ln \frac{4 \mu^{2}}{M_{s}^{2}}-1\right] \ln \frac{2 \delta}{\left|\phi_{r+1}^{+}\right|} .
\end{aligned}
$$

By the same arguments used at the end of section II in the case of a point-like four-fermion interaction, the first two equations yield $\phi_{r+}^{+} \equiv-\phi_{\ell-}^{+}$, where $\phi_{r+}^{+}$is a solution of $1=g^{2} /\left(8 \pi^{2}\right) \ln \left(2 \delta /\left|\phi_{r+}^{+}\right|\right)$. The last two equations indicate that the gap functions for the quasi-antiparticles are larger than those for the quasiparticles by a factor $\ln \left[4 \mu^{2} / M_{s}^{2}\right]-1$. In conclusion, the solution of the gap equations to leading order in weak coupling reads:

$$
\begin{aligned}
& \phi_{r+}^{+}=-\phi_{\ell-}^{+}=2 \delta \exp \left[-\frac{8 \pi^{2}}{g^{2}}\right] \sim \mu \exp \left[-\frac{8 \pi^{2}}{g^{2}}\right], \\
& \phi_{r-}^{-}=-\phi_{\ell+}^{-}=\left[\ln \frac{4 \mu^{2}}{M_{s}^{2}}-1\right] \phi_{r+}^{+} .
\end{aligned}
$$

By eq. (B35), this confirms the result already obtained in the limit $M_{s} \rightarrow \infty$ that $J=0$ pairing of fermions interacting via scalar boson exchange only occurs in the $0^{+}$channel. Note, however, that in contrast to the $M_{s} \rightarrow \infty$ case, where $\phi_{r-}^{-}=\phi_{r+}^{+}$, in weak coupling there are two independent gaps instead of one, with $\phi_{r-}^{-} \sim \ln \left(1 / g^{2}\right) \phi_{r+}^{+}$.

\section{B. Strong coupling}

In strong coupling, fermions of all energies $|\mathbf{q}|$ contribute to the functions $\mathcal{F}_{0,1}^{ \pm}$, so that they have to be computed numerically. Since these functions depend on $k \equiv|\mathbf{k}|$, the set of algebraic equations (30) is consistent only if this $k$ dependence is negligible. Before actually solving (30), we therefore investigate the 
functional dependence of $\mathcal{F}_{0,1}^{ \pm}$on $k$ in detail. This dependence is shown in Fig. 3 for $g=10$ and in Fig. 4 for $g=1$ for various values of $\phi$. The renormalization scale is $\Lambda=10 \mu$ in both cases.

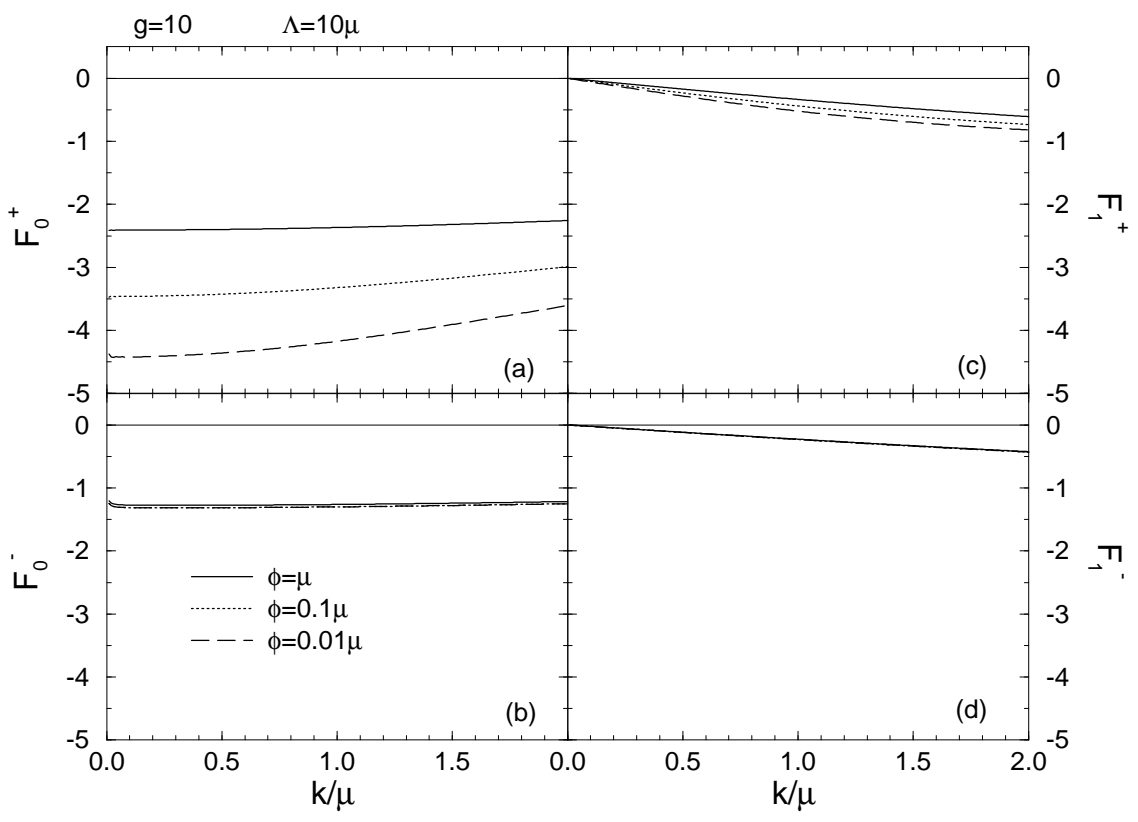

FIG. 3. The functions $\mathcal{F}_{0,1}^{ \pm}$as functions of $k / \mu$ for $\phi=\mu$ (solid), $0.1 \mu$ (dotted), and $0.01 \mu$ (dashed). The coupling constant is $g=10$, the renormalization scale $\Lambda=10 \mu$, the temperature is taken to be $T=0$.

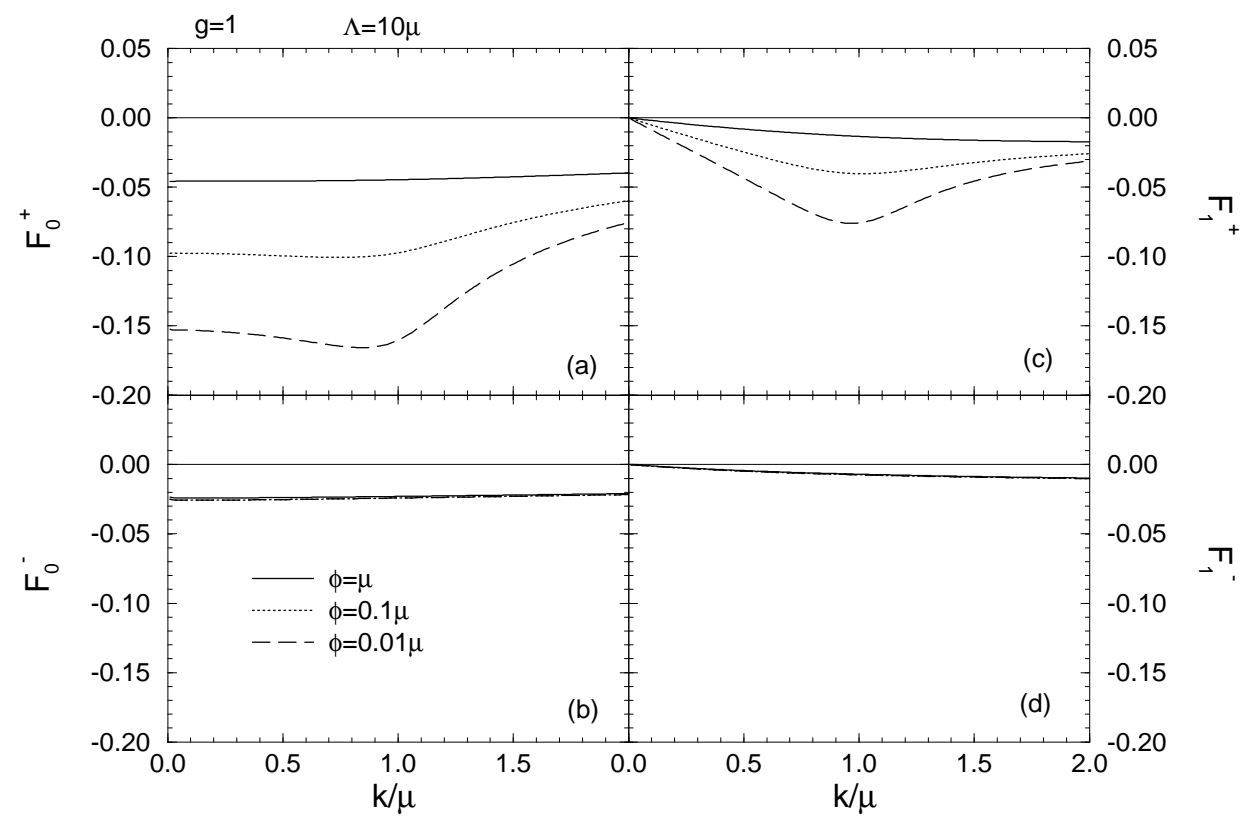

FIG. 4. Same as in Fig. 3, but for $g=1$.

As the coupling $g$ or $\phi$ become larger, the functions $\mathcal{F}_{0,1}^{ \pm}$depend less strongly on $k$. In weak coupling and for small $\phi, \mathcal{F}_{0,1}^{-}$remain approximately constant, although they are then small in magnitude, while $\mathcal{F}_{0,1}^{+}$become peaked around the Fermi surface. In strong coupling, $\mathcal{F}_{1}^{ \pm}$is smaller than $\mathcal{F}_{0}^{ \pm}$, since then 
$M_{s} \sim g \mu$ becomes large and the theory approaches the NJL limit, where $\mathcal{F}_{1}^{ \pm} \equiv 0$. Note that changes in the renormalization scale shift the values of the functions $\mathcal{F}_{0}^{ \pm}$by a constant amount; a larger $\Lambda$ increases the absolute values of these functions.

Lastly we find that while the functions $\mathcal{F}_{0,1}^{+}$change with $\phi$, the functions $\mathcal{F}_{0,1}^{-}$are nearly independent of $\phi$. The latter is easily understood for small $|\phi| \ll \mu$, since $\phi$ only enters the integrals through $\epsilon^{-}=$ $\sqrt{(q+\mu)^{2}+\phi^{2}} \simeq 2 \mu$ for $|\phi| \ll \mu$. It is interesting, however, that this behavior persists also for $|\phi|$ of order $\mu$.

In conclusion, neglecting a possible $k$ dependence does not appear to be a bad approximation unless the coupling becomes substantially smaller than 1. The results of subsection III A are then sufficient to obtain the exponential dependence of the gaps on the coupling; to determine the prefactor $\delta$ requires more sophisticated methods to solve the gap equations, for instance the approach of Ref. 20. However, the gap energies become rather small for $g<1$, cf. Fig. 5. We shall therefore numerically evaluate the gap equations (30) only for $g \geq 1$, where the $k$ dependence can be safely neglected. In what follows, we then always take the values of the functions $\mathcal{F}_{0,1}^{ \pm}$at momentum $k=\mu$.

Solving the gap equations (30) numerically, we find that the solutions satisfy the relations $\phi_{r+}^{+}=$ $-\phi_{\ell-}^{+}, \phi_{r-}^{-}=-\phi_{\ell+}^{+}$, as is true in the limits of $M_{s} \rightarrow \infty$ and weak coupling. According to eq. (B35), this implies that for massless fermions interacting via scalar boson exchange, condensation is possible only in the $0^{+}$channel.

In Fig. 5 (a) we show the dependence of the quantity $\phi \equiv \sqrt{\left[\phi_{r+}^{+}\right]^{2}+\left[\phi_{\ell-}^{+}\right]^{2}+\left[\phi_{r-}^{-}\right]^{2}+\left[\phi_{\ell+}^{-}\right]^{2}}$ at $T=0$ on $g^{2} / 4 \pi$, and in Fig. 5 (b) the associated behavior of $\phi_{r-}^{-}$and $\phi_{r+}^{+}$, normalized to $\phi$, both for different values of the renormalization scale $\Lambda$.

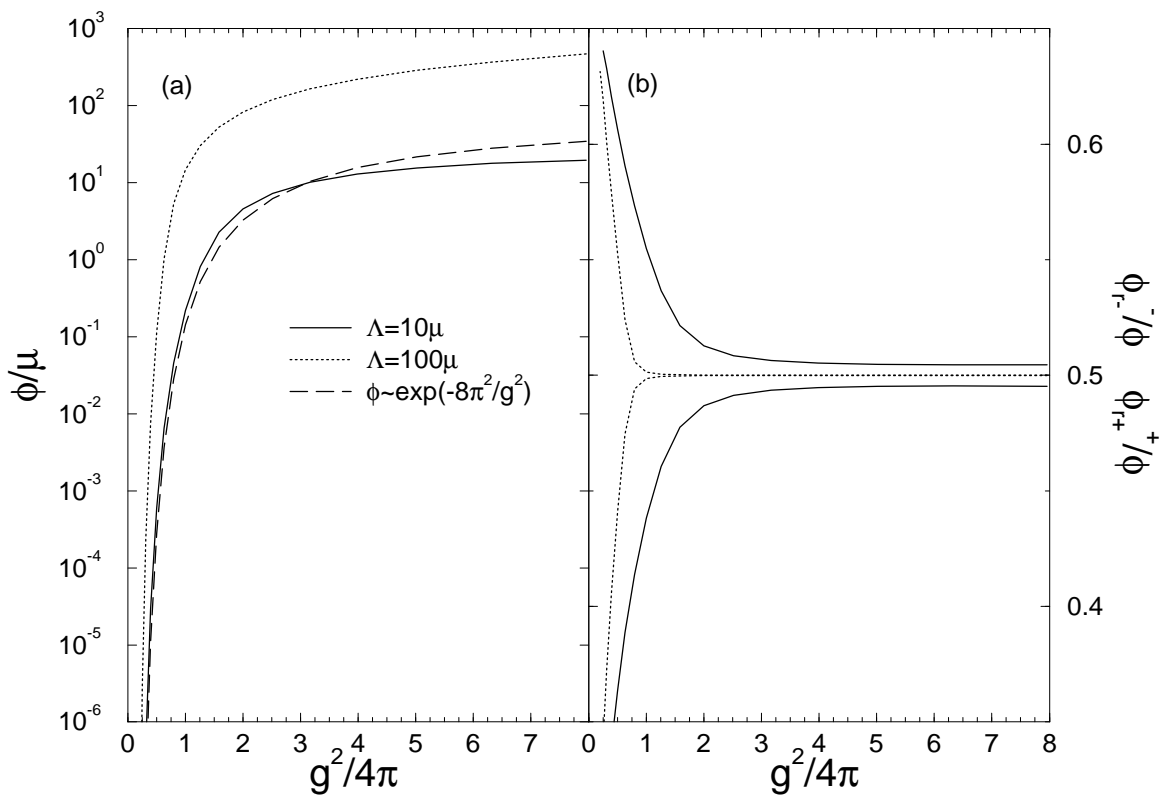

FIG. 5. (a) The dependence of $\phi$ (in units of $\mu$ ) on $g^{2} / 4 \pi$ for $\Lambda=10 \mu$ (solid) and $100 \mu$ (dotted). (b) The corresponding values of $\phi_{r-}^{-}$(upper set of curves) and $\phi_{r+}^{+}$(lower set of curves), in units of $\phi$. The dashed curve in (a) corresponds to the weak-coupling limit for $\phi$.

The value of $\phi$ decreases rapidly with the coupling, but values of $\phi$ of the order of $\mu$ and larger are possible if the coupling is sufficiently large. The value of $\phi$ increases with increasing renormalization scale $\Lambda$. The overall behavior is in good agreement with the weak-coupling limit

$$
\phi=2 \delta\left\{2+2\left(\ln \left[\frac{4 \mu^{2}}{M_{s}^{2}}\right]-1\right)^{2}\right\}^{1 / 2} \exp \left[-\frac{8 \pi^{2}}{g^{2}}\right]
$$

which is shown by the dashed line. Here, $\delta \simeq 25 \mu$ was taken to fit the numerical results for $\Lambda=10 \mu$. For a respectively larger value, the weak-coupling limit can also approximately fit the results for $\Lambda=100 \mu$. 


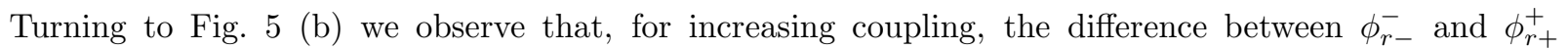
decreases. This is in accord with our expectations, since the mass increases $\sim g$, and consequently, the boson propagator approaches the form $D\left(p, M_{s}\right) \simeq 1 / M_{s}^{2}$. We have already discussed at the end of section III that in this case $\phi_{r+}^{+}=\phi_{r-}^{-}$and $\phi_{\ell-}^{+}=\phi_{\ell+}^{-}$. An interesting observation is that the values for the quasiantiparticle gap $\phi_{r-}^{-}$are larger than those for the quasiparticle gap $\phi_{r+}^{+}$for all values of $g$, not just in weak coupling, cf. eq. (40).

\section{Temperature dependence of the gap}

Finally, we discuss the temperature dependence of the gap functions. As in ordinary superconductors, the value of $\phi$ decreases with temperature, and vanishes at $T_{c}$, the critical temperature for the onset of superconductivity (or, in our case, superfluidity). $T_{c}$ can be estimated in weak coupling, where the integrals peak in a narrow region around $\mu$. With $\phi \equiv 0$ one derives

$$
\begin{aligned}
& \mathcal{F}_{0}^{+} \simeq-\frac{g^{2}}{16 \pi^{2}} \ln \frac{4 \mu^{2}}{M_{s}^{2}} \ln \frac{\zeta \delta}{T_{c}}, \\
& \mathcal{F}_{1}^{+} \simeq-\frac{g^{2}}{16 \pi^{2}}\left[\ln \frac{4 \mu^{2}}{M_{s}^{2}}-2\right] \ln \frac{\zeta \delta}{T_{c}}, \\
& \mathcal{F}_{0}^{-} \simeq 0, \quad \mathcal{F}_{1}^{-} \simeq 0,
\end{aligned}
$$

where $\zeta \equiv 2 e^{\gamma} / \pi$, and $\gamma$ is the Euler-Mascheroni constant. Using $\phi_{r+}^{+}=-\phi_{\ell-}^{+}$, the gap equations at $T_{c}$ yield the condition

$$
1=\frac{g^{2}}{8 \pi^{2}} \ln \frac{\zeta \delta}{T_{c}}
$$

for the critical temperature, i.e.,

$$
T_{c}=\frac{\zeta}{2} \phi_{r+}^{+}(T=0) \simeq 0.57 \phi_{r+}^{+}(T=0) .
$$

This relation is identical to that found in BCS theory [1].

For arbitrary $g$, the value of $T_{c}$ has to be determined numerically. For the temperature-dependent boson mass considered here, the increase of the mass with $T$ actually leads to smaller values for $T_{c}$ than expected from the BCS result (44), see Fig. 6. This is in agreement with the general expectation that larger boson masses lead to smaller values for the gap $\phi$, and consequently to smaller values of $T_{c}$. We find that $\phi_{r+}^{+}$and $\phi_{r-}^{-}$always vanish at the same temperature for any value of the coupling. 


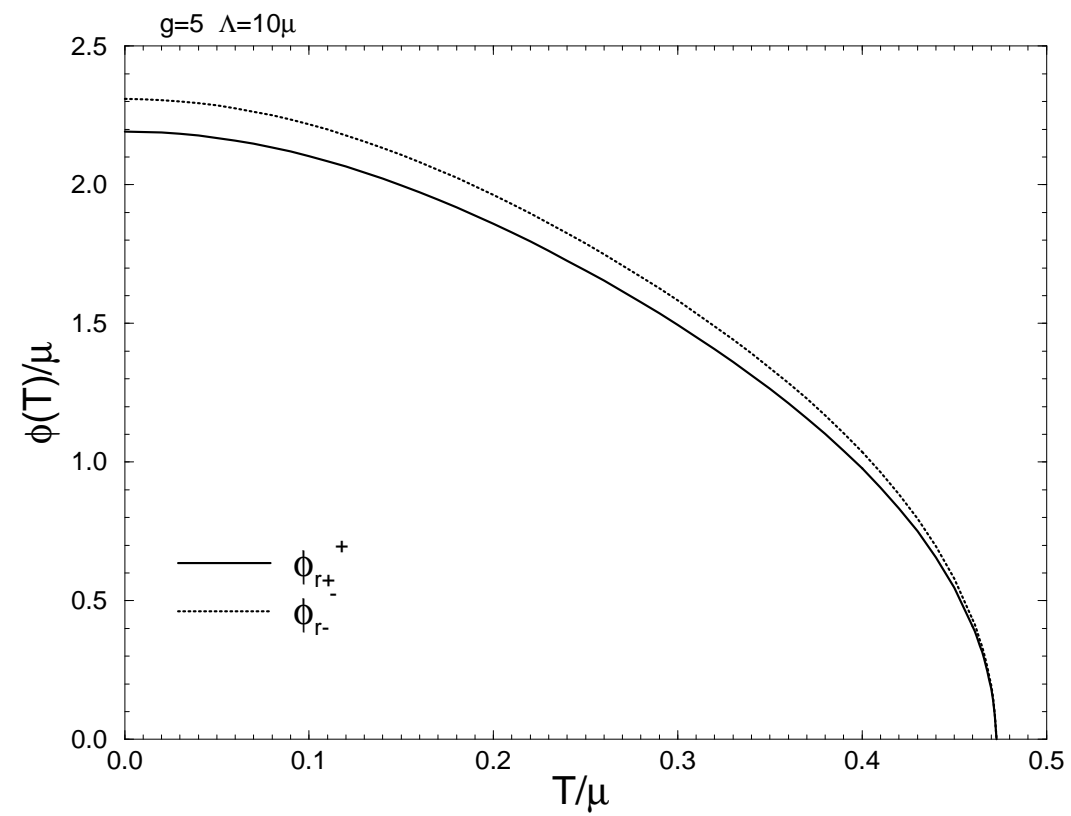

FIG. 6. The temperature dependence of $\phi_{r+}^{+}$and $\phi_{r-}^{-}$for $g=5, \Lambda=10 \mu$. $T_{c} \simeq 0.47 \mu$ is smaller than the value $0.57 \phi_{r+}^{+}(T=0) \simeq 1.25 \mu$ expected in weak coupling.

\section{CONCLUSIONS}

In this paper, we have investigated superfluidity in a system of massless fermions interacting via scalar boson exchange. The gap matrix contains in general four independent gap functions, corresponding to the condensation of fermions with the same helicity and chirality. We solved the gap equations in the mean-field approximation as functions of the coupling $g$ and the renormalization scale $\Lambda$. For scalar boson exchange, condensation in the $0^{-}$channel does not occur, and the number of independent gap functions reduces to two, one for quasiparticles and one for quasi-antiparticles. The quasi-antiparticle gap is found to be larger than the quasiparticle gap, by a factor $\sim \ln \left(1 / g^{2}\right)$ in weak coupling. This is in contrast to NJL-type models, where the point-like four-fermion interactions do not allow for pairing with $L=S=1$ and force the equality of the quasiparticle and quasi-antiparticle gaps. We also analyzed the temperature dependence of the gap functions, and found that a temperature-dependent boson mass can significantly reduce $T_{c}$.

Qualitatively, our model shares the feature with QCD that the gap is exponentially small for $g \ll 1$. As can be seen from Fig. 5 (a), in that regime small variations of the coupling lead to order-of-magnitude changes in the gap. Moreover, the gap energies are quite sensitive to the value of the ultraviolet cut-off $\Lambda_{\mathrm{UV}} \sim \Lambda$. Taking a small value for the coupling, Bailin and Love found gap energies which are extremely small, $\sim 10^{-3} \mu \sim 1 \mathrm{MeV}$ for $\mu \sim 1 \mathrm{GeV}$ [3]. In more recent studies 6, 6, 9, 13], much larger gaps, $\sim 100$ $\mathrm{MeV}$, were found. Such large gaps can be obtained from the treatment of Bailin and Love simply by taking larger coupling constants. Alternatively, the latter studies would get smaller values for the gap by reducing the coupling constant, or changing the form factors employed there to remove the ultraviolet divergence of the gap integrals.

Clearly, it is essential to correctly compute the magnitude of the gap in QCD. Studies along this line are in progress 17, 18].

\section{Acknowledgments}

We acknowledge discussions with M. Alford, A. Blaer, D. Blaschke, V.J. Emery, M. Gyulassy, M. Laine, C. Manuel, B. Müller, V.N. Muthukumar, K. Rajagopal, T. Schäfer, and D.T. Son. D.H.R. thanks Columbia University's Nuclear Theory Group for continuing access to their computing facilities. 


\section{APPENDIX A: THE MEAN-FIELD APPROXIMATION}

Consider a statistical mechanical system at finite temperature $T$ and chemical potential $\mu$ where fermions interact via exchange of an $N$-component bosonic field. For $N=1$ the Lagrangian is identical with eq. (1). The grand partition function of this system is

$$
\begin{aligned}
\mathcal{Z} & =\mathcal{N} \int \mathcal{D} \bar{\psi} \mathcal{D} \psi \mathcal{D} \phi \exp \{I[\bar{\psi}, \psi, \phi]\} \\
I[\bar{\psi}, \psi, \phi] & =\int_{x, y}\left(\bar{\psi}(x)\left[G_{0}^{+}\right]^{-1}(x, y) \psi(y)-\frac{1}{2} \sum_{a, b=1}^{N} \phi^{a}(x) D_{a b}^{-1}(x, y) \phi^{b}(y)\right) \\
& -\int_{x} \sum_{a=1}^{N} g \bar{\psi}(x) \Gamma_{a} \psi(x) \phi^{a}(x) .
\end{aligned}
$$

Here,

$$
\left[G_{0}^{ \pm}\right]^{-1}(x, y) \equiv-i\left[i \gamma \cdot \partial \pm \mu \gamma_{0}-m\right] \delta^{(4)}(x-y) .
$$

$D_{a b}(x, y)$ is the boson propagator, the structure of which need not be further specified at this point. The above form includes scalar interactions for $N=1, \Gamma_{1}=1$, vector interactions for $N=4, \phi^{a}=g^{a-1, \mu} \phi_{\mu}$, $\Gamma_{a}=g_{a-1, \mu} \gamma^{\mu}, D_{a b}^{-1}=g_{a-1, \mu}\left[D^{-1}\right]^{\mu \nu} g_{b-1, \nu}, \mu, \nu=0, \ldots, 3$, and similarly for other interactions. The bosonic fields can be formally integrated out with the result

$$
\begin{aligned}
\mathcal{Z} & =\mathcal{N}^{\prime}\left(\operatorname{det} D^{-1}\right)^{-1 / 2} \int \mathcal{D} \bar{\psi} \mathcal{D} \psi \exp \{I[\bar{\psi}, \psi]\} \\
I[\bar{\psi}, \psi] & =\int_{x, y}\left(\bar{\psi}(x)\left[G_{0}^{+}\right]^{-1}(x, y) \psi(y)+\frac{g^{2}}{2} \sum_{a, b} \bar{\psi}(x) \Gamma_{a} \psi(x) D^{a b}(x, y) \bar{\psi}(y) \Gamma_{b} \psi(y)\right) .
\end{aligned}
$$

The last term physically corresponds to the current-current interaction displayed in Fig. 月. Since it is biquadratic in the fermion fields, the integration over $\bar{\psi}, \psi$ cannot be carried out. In this Appendix, we discuss mean-field approximations to (A3a). The idea is to approximate the biquadratic term in (A3b) by a bilinear term times a fermion condensate, which then allows for integrating over $\bar{\psi}, \psi$. In principle, one can either have an antifermion-fermion condensate or a fermion-fermion condensate, the latter being the case of interest in describing the phenomenon of superconductivity.

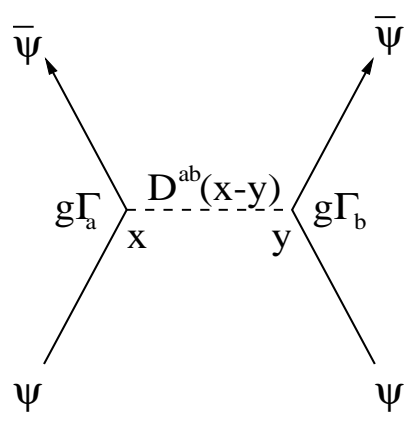

FIG. 7. The current-current interaction.

\section{The mean-field approximation for an antifermion-fermion condensate}

In the mean-field approximation one approximates two of the four fermion fields in the last term in (A3b) by their expectation value in order to obtain a bilinear form in the fermion fields which allows for integration over $\bar{\psi}, \psi$ in (A3a). One possibility is to contract $\bar{\psi}$ and $\psi$, as shown in Fig. 8 (a). The expectation value $\langle\bar{\psi} \psi\rangle$ corresponds to an antifermion-fermion condensate. 
More specifically [21, define

$$
j_{a}(x) \equiv g \bar{\psi}(x) \Gamma_{a} \psi(x),
$$

and introduce the fluctuation $\rho_{a}(x)$ of $j_{a}(x)$ around its expectation value $\left\langle j_{a}(x)\right\rangle$,

$$
\rho_{a}(x)=j_{a}(x)-\left\langle j_{a}(x)\right\rangle .
$$

Then, assuming $D_{a b}(x, y)=D_{b a}(y, x)$, one has to first order in $\rho_{a}$ :

$$
\begin{aligned}
& \frac{g^{2}}{2} \sum_{a, b} \bar{\psi}(x) \Gamma_{a} \psi(x) D^{a b}(x, y) \bar{\psi}(y) \Gamma_{b} \psi(y) \equiv \frac{1}{2} \sum_{a, b} j_{a}(x) D^{a b}(x, y) j_{b}(y) \\
& \simeq \frac{1}{2} \sum_{a, b}\left[\left\langle j_{a}(x)\right\rangle D^{a b}(x, y)\left\langle j_{b}(y)\right\rangle+2 \rho_{a}(x) D^{a b}(x, y)\left\langle j_{b}(y)\right\rangle\right] \\
& =\frac{1}{2} \sum_{a, b}\left[-\left\langle j_{a}(x)\right\rangle D^{a b}(x, y)\left\langle j_{b}(y)\right\rangle+2 j_{a}(x) D^{a b}(x, y)\left\langle j_{b}(y)\right\rangle\right] .
\end{aligned}
$$

Inserting this back into (A3b) and integrating over the fermion fields in (A3a) gives the partition function in the mean-field approximation for an antifermion-fermion condensate

$$
\mathcal{Z}_{\langle\overline{\mathrm{F} F}\rangle}=\mathcal{N}^{\prime}\left(\operatorname{det} D^{-1}\right)^{-1 / 2} \operatorname{det}\left[G_{\langle\overline{\mathrm{F} F}\rangle}^{+}\right]^{-1} \exp \left\{-\frac{g^{2}}{2} \int_{x, y} \sum_{a, b}\left\langle\bar{\psi}(x) \Gamma_{a} \psi(x)\right\rangle D^{a b}(x, y)\left\langle\bar{\psi}(y) \Gamma_{b} \psi(y)\right\rangle\right\}
$$

where the (inverse) fermion propagator is

$$
\left[G_{\langle\overline{\mathrm{F} F}\rangle}^{+}\right]^{-1}(x, y) \equiv\left[G_{0}^{+}\right]^{-1}(x, y)-i g^{2} \delta^{(4)}(x-y) \int_{z} \sum_{a, b} \Gamma_{a} D^{a b}(x, z)\left\langle\bar{\psi}(z) \Gamma_{b} \psi(z)\right\rangle
$$

This last equation is obviously a self-consistency equation (or gap equation, or Schwinger-Dyson equation) for the mean-field propagator, since

$$
\left\langle\bar{\psi}(z) \Gamma_{b} \psi(z)\right\rangle \equiv \operatorname{Tr}\left\{G_{\langle\overline{\mathrm{F}} \mathrm{F}\rangle}^{+}(z, z) \Gamma_{b}\right\} .
$$

The explicit evaluation of the partition function is facilitated assuming translational invariance which allows to Fourier-transform all quantities to momentum space, for details, see [21].

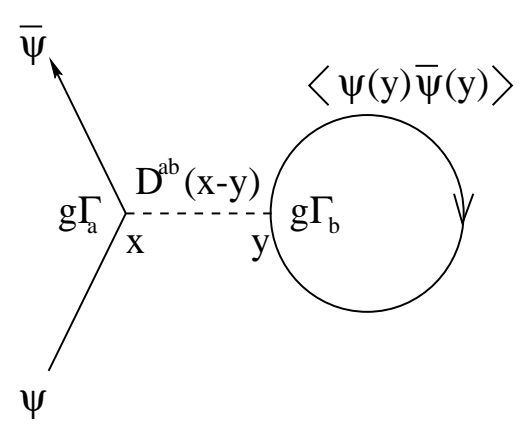

(a)

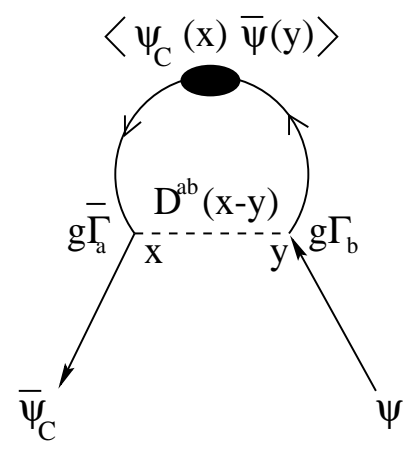

(b)

FIG. 8. (a) The mean-field approximation for an antifermion-fermion condensate, obtained by contracting $\bar{\psi}(y)$ and $\psi(y)$. (b) The mean-field approximation for a fermion-fermion condensate, contracting $\bar{\psi}(x)$ and $\bar{\psi}(y)$. 


\section{The mean-field approximation for a fermion-fermion condensate}

In the previous subsection A 1, we have discussed the mean-field approximation for an antifermion-fermion condensate. Now we discuss condensation of fermion pairs. Instead of contracting $\bar{\psi}$ and $\psi$, we now contract $\bar{\psi}$ and $\bar{\psi}$ (or equivalently, $\psi$ and $\psi$ ), cf. Fig. 8 (b). This leads to a fermion-fermion condensate. More specifically, introduce the charge-conjugate spinor $\psi_{C}$ via (3), and define $\bar{\Gamma}_{a}$ as

$$
\bar{\Gamma}_{a}=C \Gamma_{a}^{T} C^{-1}
$$

Then, the four-fermion term in (A3b) is written as

$$
\begin{aligned}
\bar{\psi}(x) \Gamma_{a} \psi(x) \bar{\psi}(y) \Gamma_{b} \psi(y) & =\frac{1}{2}\left[\bar{\psi}_{C}(x) \bar{\Gamma}_{a} \psi_{C}(x) \bar{\psi}(y) \Gamma_{b} \psi(y)+\text { h.c. }\right] \\
& =-\frac{1}{2} \operatorname{Tr}\left[\gamma_{0} J^{\dagger}(y, x) \gamma_{0} \bar{\Gamma}_{a} J(x, y) \Gamma_{b}+\text { h.c. }\right]
\end{aligned}
$$

where we introduced the $4 \times 4$ matrix

$$
J_{\alpha \beta}(x, y) \equiv \psi_{C \alpha}(x) \bar{\psi}_{\beta}(y), \quad J_{\beta \alpha}^{\dagger}(y, x)=\left[J_{\alpha \beta}(x, y)\right]^{\dagger}=\left[\gamma_{0} \psi(y)\right]_{\beta}\left[\bar{\psi}_{C}(x) \gamma_{0}\right]_{\alpha} .
$$

Introducing the fluctuation of this matrix around its expectation value,

$$
\rho(x, y) \equiv J(x, y)-\langle J(x, y)\rangle
$$

and expanding A11 to linear order in $\rho$ yields

$$
\begin{aligned}
& \bar{\psi}(x) \Gamma_{a} \psi(x) \bar{\psi}(y) \Gamma_{b} \psi(y) \\
& \simeq \frac{1}{2} \operatorname{Tr}\left[\gamma_{0}\left\langle J^{\dagger}(y, x)\right\rangle \gamma_{0} \bar{\Gamma}_{a}\langle J(x, y)\rangle \Gamma_{b}-2 \gamma_{0} J^{\dagger}(y, x) \gamma_{0} \bar{\Gamma}_{a}\langle J(x, y)\rangle \Gamma_{b}+\text { h.c. }\right] .
\end{aligned}
$$

The result for the partition function in the mean-field approximation for a fermion-fermion condensate reads

$$
\begin{aligned}
\mathcal{Z}_{\langle\mathrm{FF}\rangle} & =\mathcal{N}^{\prime}\left(\operatorname{det} D^{-1}\right)^{-1 / 2} \exp \left\{\frac{g^{2}}{4} \int_{x, y} \sum_{a, b} \operatorname{Tr}\left[\gamma_{0}\left\langle J^{\dagger}(y, x)\right\rangle \gamma_{0} \bar{\Gamma}_{a}\langle J(x, y)\rangle \Gamma_{b}+\text { h.c. }\right] D^{a b}(x, y)\right\} \\
& \times \int \mathcal{D} \bar{\psi} \mathcal{D} \psi \exp \{I[\bar{\psi}, \psi]\}
\end{aligned}
$$

with

$$
I[\bar{\psi}, \psi]=\int_{x, y}\left\{\bar{\psi}(x)\left[G_{0}^{+}\right]^{-1}(x, y) \psi(y)+\frac{1}{2}\left[\bar{\psi}_{C}(x) \Delta^{+}(x, y) \psi(y)+\text { h.c. }\right]\right\}
$$

where

$$
\Delta^{+}(x, y) \equiv g^{2} \sum_{a, b} \bar{\Gamma}_{a}\left\langle\psi_{C}(x) \bar{\psi}(y)\right\rangle \Gamma_{b} D^{a b}(x, y)
$$

This equation uniquely defines the gap equation in the mean-field approximation. In order to solve this equation, one has to compute the expectation value $\left\langle\psi_{C}(x) \bar{\psi}(y)\right\rangle$. This is done as follows. The hermitean conjugate of the first term in square brackets in (A16) is

$$
\left[\bar{\psi}_{C}(x) \Delta^{+}(x, y) \psi(y)\right]^{\dagger}=\bar{\psi}(y) \Delta^{-}(y, x) \psi_{C}(x)
$$

where

$$
\Delta^{-}(y, x) \equiv \gamma_{0}\left[\Delta^{+}(x, y)\right]^{\dagger} \gamma_{0}
$$

The first term in A16 can be rewritten in terms of charge-conjugate spinors as 


$$
\bar{\psi}(x)\left[G_{0}^{+}\right]^{-1}(x, y) \psi(y)=\bar{\psi}_{C}(y)\left[G_{0}^{-}\right]^{-1}(y, x) \psi_{C}(x) .
$$

Introducing the 8-component spinors

$$
\Psi \equiv\left(\begin{array}{c}
\psi \\
\psi_{C}
\end{array}\right), \quad \bar{\Psi} \equiv\left(\bar{\psi}, \bar{\psi}_{C}\right),
$$

the action (A16) can be written in compact matrix notation as

$$
I[\bar{\Psi}, \Psi]=\frac{1}{2} \int_{x, y} \bar{\Psi}(x) \mathcal{S}^{-1}(x, y) \Psi(y),
$$

where

$$
\mathcal{S}^{-1}=\left(\begin{array}{cc}
{\left[G_{0}^{+}\right]^{-1}} & \Delta^{-} \\
\Delta^{+} & {\left[G_{0}^{-}\right]^{-1}}
\end{array}\right) .
$$

Let us assume translational invariance for the gap matrix, $\Delta^{ \pm}(x, y)=\Delta^{ \pm}(x-y)$. Then, the Fourier transforms of the fields and $\left[G_{0}^{ \pm}\right]^{-1}, \Delta^{ \pm}$are

$$
\begin{array}{r}
\psi(x)=\frac{1}{\sqrt{V}} \sum_{k} e^{-i k \cdot x} \psi(k), \bar{\psi}(x)=\frac{1}{\sqrt{V}} \sum_{k} e^{i k \cdot x} \bar{\psi}(k), \\
\psi_{C}(x)=\frac{1}{\sqrt{V}} \sum_{k} e^{-i k \cdot x} \psi_{C}(k), \bar{\psi}_{C}(x)=\frac{1}{\sqrt{V}} \sum_{k} e^{i k \cdot x} \bar{\psi}_{C}(k), \\
{\left[G_{0}^{ \pm}\right]^{-1}(x)=\frac{T}{V} \sum_{k} e^{-i k \cdot x}\left[G_{0}^{ \pm}\right]^{-1}(k), \Delta^{ \pm}(x)=\frac{T}{V} \sum_{k} e^{-i k \cdot x} \Delta^{ \pm}(k) .}
\end{array}
$$

Note the choice of signs in the exponential factors for $\psi_{C}$ and $\Delta^{-}$. From eq. (3), one would have expected the opposite sign. This choice has the consequence

$$
\psi_{C}(k)=C \bar{\psi}^{T}(-k), \quad \bar{\psi}_{C}(k)=\psi^{T}(-k) C, \quad \Delta^{-}(k)=\gamma^{0}\left[\Delta^{+}(k)\right]^{\dagger} \gamma^{0} .
$$

It also ensures that the action is diagonal in momentum space:

$$
I[\bar{\Psi}, \Psi]=\frac{1}{2} \sum_{k} \bar{\Psi}(k) \frac{\mathcal{S}^{-1}(k)}{T} \Psi(k) .
$$

In the conventional approach to superconductivity, the action is only diagonalized after performing a Bogoliubov transformation. The choice of signs in the Fourier transforms (A24b, A24c for the charge-conjugate spinor and $\Delta^{-}$avoids this additional complication.

In order to complete the calculation of the grand partition function in the mean-field approximation, one has to perform the Grassmann integration over the fermion fields $\bar{\psi}, \psi$. In the action (A26), however, the fields $\psi_{C}(k), \bar{\psi}_{C}(k)$ also enter; these are not independent integration variables on account of (3). To proceed, from $(\mathrm{A} 25)$ one derives the identities

$$
\psi(-k) \equiv C \bar{\psi}_{C}^{T}(k) \quad, \quad \bar{\psi}(-k) \equiv \psi_{C}^{T}(k) C,
$$

and rewrites the integration measure as

$$
\mathcal{D} \bar{\psi} \mathcal{D} \psi \equiv \prod_{k} \mathrm{~d} \bar{\psi}(k) \mathrm{d} \psi(k)=\prod_{k>0} \mathrm{~d} \bar{\psi}(k) \mathrm{d} \bar{\psi}(-k) \mathrm{d} \psi(k) \mathrm{d} \psi(-k)=\tilde{\mathcal{N}} \prod_{k>0} \mathrm{~d} \bar{\psi}(k) \mathrm{d} \psi_{C}(k) \mathrm{d} \psi(k) \mathrm{d} \bar{\psi}_{C}(k),
$$

where $\tilde{\mathcal{N}}$ is the (irrelevant, since constant) Jacobian from the transformation A27). Moreover, one can show that

$$
\frac{1}{2} \sum_{k} \bar{\Psi}(k) \frac{\mathcal{S}^{-1}(k)}{T} \Psi(k) \equiv \sum_{k>0} \bar{\Psi}(k) \frac{\mathcal{S}^{-1}(k)}{T} \Psi(k) .
$$


Then,

$$
\int \mathcal{D} \bar{\psi} \mathcal{D} \psi \exp \{I[\bar{\Psi}, \Psi]\} \equiv \tilde{\mathcal{N}} \operatorname{det}_{k>0}\left[\frac{\mathcal{S}^{-1}}{T}\right] \equiv \tilde{\mathcal{N}}\left(\operatorname{det}\left[\frac{\mathcal{S}^{-1}}{T}\right]\right)^{1 / 2}
$$

The full propagator $\mathcal{S}(k)$ is determined from solving $1=\mathcal{S}^{-1} \mathcal{S}$, with the result

$$
\mathcal{S}=\left(\begin{array}{cc}
G^{+} & -G_{0}^{+} \Delta^{-} G^{-} \\
-G_{0}^{-} \Delta^{+} G^{+} & G^{-}
\end{array}\right)
$$

where all functions depend on the 4-momentum $k^{\mu}$ and where we have introduced

$$
G^{ \pm} \equiv\left\{\left[G_{0}^{ \pm}\right]^{-1}-\Sigma^{ \pm}\right\}^{-1}, \quad \Sigma^{ \pm} \equiv \Delta^{\mp} G_{0}^{\mp} \Delta^{ \pm} .
$$

The off-diagonal components of $\mathcal{S}$ fulfill the identity

$$
G_{0}^{\mp} \Delta^{ \pm} G^{ \pm}=G^{\mp} \Delta^{ \pm} G_{0}^{ \pm} .
$$

This can be proven directly, or by solving $1=\mathcal{S S}^{-1}$.

From eq. (A31), and from $\left\langle\Psi_{\alpha}(k) \bar{\Psi}_{\beta}(k)\right\rangle \equiv-T \mathcal{S}_{\alpha \beta}(k)$ (this identity is proven e.g. in Appendix B of [21]) one obtains

$$
\left\langle\psi_{C}(x) \bar{\psi}(y)\right\rangle \equiv \frac{T}{V} \sum_{k} e^{-i k \cdot(x-y)} G_{0}^{-}(k) \Delta^{+}(k) G^{+}(k) .
$$

Inserting this into (A17) and taking the Fourier transform, one obtains the gap equation

$$
\Delta^{+}(k)=g^{2} \frac{T}{V} \sum_{q} \sum_{a, b} \bar{\Gamma}_{a} D^{a b}(k-q) G_{0}^{-}(q) \Delta^{+}(q) G^{+}(q) \Gamma_{b} .
$$

The gap equation has a graphical representation which is derived from Fig. 8(b), cf. Fig. 9. All that changed with respect to Fig. 8 (b) is that the explicit value of $\left\langle\psi_{C}(x) \bar{\psi}(y)\right\rangle$ from (A31) was used. The blob in Fig. 9 stands for the gap matrix, while the thick (thin) lines represent the full (free) propagator.

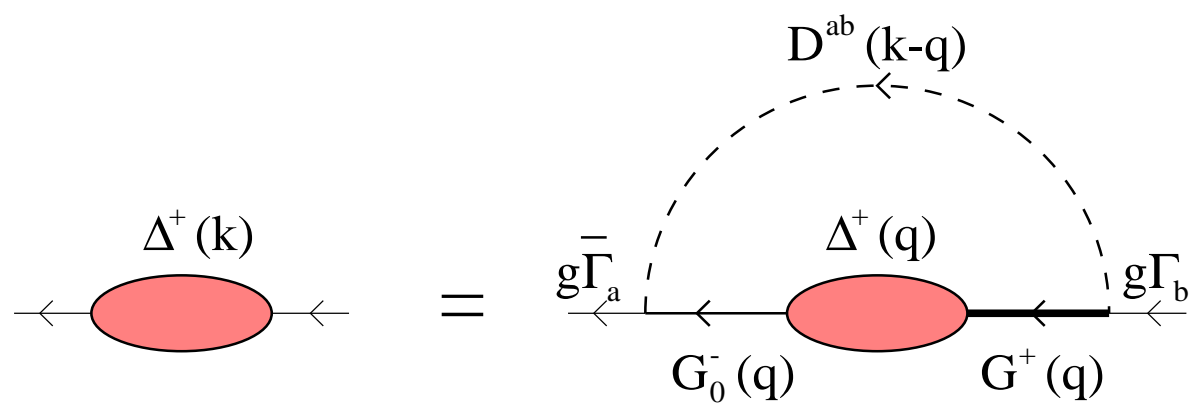

FIG. 9. The gap equation.

\section{APPENDIX B: THE STRUCTURE OF A SCALAR GAP MATRIX}

In this Appendix we analyze the Dirac structure of a scalar gap matrix. We find that in general there are eight independent gap functions. Fermi statistics imposes a powerful symmetry constraint on these functions. With respect to parity, four of the gap functions describe condensation in the channel of even parity, the other four in the odd-parity channel. Only fermions with the same helicity condense. With respect to chirality, four gap functions describe condensation of pairs with the same, the other four that of pairs with the opposite chirality. The chirality and helicity properties of the gap functions suggest a different representation scheme for the gap matrix. Within this scheme, we derive that the number of independent gap functions reduces to four in the ultrarelativistic limit. 


\section{Dirac structure}

The Fourier transform $\Delta(k)$ of the (translationally invariant) scalar gap matrix $\Delta(x-y)$ can be expanded in the basis of the 16 linearly independent $4 \times 4$ matrices. However, for condensation in the $J=0$ channel, all Lorentz indices have to be contracted with either the 4-momentum, $k^{\mu}$, or, because of the presence of a medium, with the respective 4 -velocity of this medium, $u^{\mu}$. This leaves the eight matrices

$$
\mathbf{1}, \gamma \cdot k, \gamma \cdot u, \gamma \cdot k \gamma \cdot u, \gamma_{5}, \gamma_{5} \gamma \cdot k, \gamma_{5} \gamma \cdot u, \gamma_{5} \gamma \cdot k \gamma \cdot u
$$

In the rest frame of the medium, $u^{\mu}=(1, \mathbf{0})$, the most general ansatz for $\Delta$ is then

$$
\Delta=\Delta_{1} \gamma_{5}+\Delta_{2} \gamma \cdot \hat{\mathbf{k}} \gamma_{0} \gamma_{5}+\Delta_{3} \gamma_{0} \gamma_{5}+\Delta_{4}+\Delta_{5} \gamma \cdot \hat{\mathbf{k}} \gamma_{0}+\Delta_{6} \gamma \cdot \hat{\mathbf{k}}+\Delta_{7} \gamma \cdot \hat{\mathbf{k}} \gamma_{5}+\Delta_{8} \gamma_{0}
$$

where $\hat{\mathbf{k}} \equiv \mathbf{k} /|\mathbf{k}|$, and where the gap matrix $\Delta$ as well as the gap functions $\Delta_{n}$ depend on $k_{0}$, $\mathbf{k}$. The notation follows that of Bailin and Love [3].

\section{Symmetry properties}

The antisymmetry of the fermion fields provides a powerful constraint on the gap functions $\Delta_{n}$. With (3) and $C=-C^{-1}$ one rewrites

$$
\int_{x, y} \bar{\psi}_{C}(x) \Delta(x-y) \psi(y)=-\int_{y, x} \psi^{T}(x)[\Delta(y-x)]^{T} \bar{\psi}_{C}^{T}(y)=\int_{y, x} \bar{\psi}_{C}(x) C^{-1}[\Delta(y-x)]^{T} C \psi(y),
$$

i.e., in Fourier space

$$
C \Delta(k) C^{-1}=\Delta^{T}(-k)
$$

Using $C \gamma_{\mu} C^{-1}=-\gamma_{\mu}^{T}$, this implies:

$$
\Delta_{n}(k)=+\Delta_{n}^{T}(-k), n=1, \ldots, 6, \Delta_{n}(k)=-\Delta_{n}^{T}(-k), n=7,8 .
$$

If we neglect the energy-momentum dependence of the gap functions $\Delta_{n}$, as we do in our solution of the gap equations, then this equation demands that $\Delta_{1}, \ldots, \Delta_{6}$ are symmetric matrices in the space of internal degrees of freedom, while $\Delta_{7}$ and $\Delta_{8}$ are antisymmetric. In QCD, for example, $\Delta_{n, f g}^{i j}=\Delta_{n, g f}^{j i}, n=1, \ldots, 6$, while $\Delta_{n, f g}^{i j}=-\Delta_{n, g f}^{j i}, n=7,8$ [for notation see the discussion preceding eq. (11)]. If the $\Delta_{n}$ do not have internal degrees of freedom, then $\Delta_{7}$ and $\Delta_{8}$ must vanish, as noted by Bailin and Love [3]. We stress, however, that $\Delta_{7}$ and $\Delta_{8}$ need not vanish when they carry internal degrees of freedom.

\section{Parity}

Under a parity transformation, $t \rightarrow t, \mathbf{x} \rightarrow-\mathbf{x}$, so 3 -vectors change their sign. On Dirac spinors, a parity transformation is effected by 22] $S(P)=\eta_{P} \gamma_{0}$, where $\eta_{P}= \pm 1$ is the intrinsic parity of a particle. Thus $\gamma_{0}$ has even parity, $S^{-1}(P) \gamma_{0} S(P)=\gamma_{0}$, while $\gamma^{i}$ has odd parity, $S^{-1}(P) \gamma^{i} S(P)=-\gamma^{i}$. Wave functions transform as $\psi^{\prime}(t, \mathbf{x})=S(P) \psi(t,-\mathbf{x})=\eta_{P} \gamma_{0} \psi(t,-\mathbf{x})$, i.e., with $(3), \bar{\psi}_{C}^{\prime}(t, \mathbf{x})=-\bar{\psi}_{C}(t,-\mathbf{x}) S(P)$, which shows that the spinor $\bar{\psi}_{C}$ has opposite parity from $\psi$.

We rewrite the action (2) in Fourier space, with the conventions listed in eq. (A24):

$$
I[\bar{\psi}, \psi]=\sum_{k}\left\{\bar{\psi}(k)\left[G_{0}^{+}\right]^{-1}(k) \psi(k)+\frac{1}{2}\left[\bar{\psi}_{C}(k) \Delta(k) \psi(k)+\text { h.c. }\right]\right\} .
$$

Then, the term

$$
\bar{\psi}_{C}(k)\left(\Delta_{1} \gamma_{5}+\Delta_{2} \gamma \cdot \hat{\mathbf{k}} \gamma_{0} \gamma_{5}+\Delta_{3} \gamma_{0} \gamma_{5}+\Delta_{7} \gamma \cdot \hat{\mathbf{k}} \gamma_{5}\right) \psi(k)
$$

represents condensation in the even-parity channel, while the term

$$
\bar{\psi}_{C}(k)\left(\Delta_{4}+\Delta_{5} \gamma \cdot \hat{\mathbf{k}} \gamma_{0}+\Delta_{6} \gamma \cdot \hat{\mathbf{k}}+\Delta_{8} \gamma_{0}\right) \psi(k)
$$

represents condensation in the odd-parity channel. 


\section{Helicity}

The spinors $\psi, \bar{\psi}, \psi_{C}, \bar{\psi}_{C}$ in the effective action $(\overline{\mathrm{B} 6})$ can be decomposed with respect to their helicity. The helicity projector is given by

$$
\mathcal{P}_{ \pm}(\mathbf{k}) \equiv \frac{1 \pm \gamma_{5} \gamma_{0} \gamma \cdot \mathbf{k}}{2}
$$

and we denote the helicity-projected spinors by

$$
\begin{array}{cl}
\psi_{ \pm}(k)=\mathcal{P}_{ \pm}(\mathbf{k}) \psi(k), & \bar{\psi}_{ \pm}(k)=\bar{\psi}(k) \mathcal{P}_{ \pm}(\mathbf{k}) \\
\psi_{C \pm}(k)=\mathcal{P}_{ \pm}(\mathbf{k}) \psi_{C}(k), & \bar{\psi}_{C \pm}(k)=\bar{\psi}_{C}(k) \mathcal{P}_{ \pm}(\mathbf{k}) .
\end{array}
$$

The second equation results from the fact that due to our sign convention in (A24b), in Fourier space $\bar{\psi}_{C}(k)=\psi^{T}(-k) C$.

The inverse free fermion propagator as well as the gap matrix (B2) commute with the helicity projector,

$$
\left[\left[G_{0}^{+}\right]^{-1}(k), \mathcal{P}_{ \pm}(\mathbf{k})\right]=\left[\Delta(k), \mathcal{P}_{ \pm}(\mathbf{k})\right]=0 .
$$

This means that the action (B6) does not mix states of different helicity,

$$
I[\bar{\psi}, \psi] \equiv \sum_{s= \pm} I\left[\bar{\psi}_{s}, \psi_{s}\right]
$$

As a consequence, condensation in a scalar $(J=0)$ channel can only occur between fermions of the same helicity $(++$ or --$)$. For a physical explanation, see the discussion following eq. (10).

\section{Chirality}

The chirality projector is given by

$$
\mathcal{P}_{r, \ell} \equiv \frac{1 \pm \gamma_{5}}{2}
$$

Let us introduce right- and left-handed spinors via

$$
\begin{array}{ll}
\psi_{r} \equiv \mathcal{P}_{r} \psi & , \quad \psi_{\ell} \equiv \mathcal{P}_{\ell} \psi, \\
\bar{\psi}_{r} \equiv \bar{\psi} \mathcal{P}_{\ell}, & \bar{\psi}_{\ell} \equiv \bar{\psi} \mathcal{P}_{r} .
\end{array}
$$

With (3), one then derives

$$
\begin{array}{ll}
\psi_{C r} \equiv \mathcal{P}_{\ell} \psi_{C} \quad, \quad \psi_{C \ell} \equiv \mathcal{P}_{r} \psi_{C} \\
\bar{\psi}_{C r} \equiv \bar{\psi}_{C} \mathcal{P}_{r} \quad, \quad \bar{\psi}_{C \ell} \equiv \bar{\psi}_{C} \mathcal{P}_{\ell} .
\end{array}
$$

Let us now investigate the effect of the chirality projectors on the gap matrix (B2). From $\gamma^{\mu} \gamma_{5}=-\gamma_{5} \gamma^{\mu}$ and $\mathcal{P}_{r, \ell}^{2}=\mathcal{P}_{r, \ell}, \mathcal{P}_{r} \mathcal{P}_{\ell}=0$ one derives

$$
\mathcal{P}_{r, \ell} \Delta \mathcal{P}_{r, \ell}=\Delta_{1} \gamma_{5}+\Delta_{2} \gamma \cdot \hat{\mathbf{k}} \gamma_{0} \gamma_{5}+\Delta_{4}+\Delta_{5} \gamma \cdot \hat{\mathbf{k}} \gamma_{0}
$$

while

$$
\mathcal{P}_{\ell, r} \Delta \mathcal{P}_{r, \ell}=\Delta_{3} \gamma_{0} \gamma_{5}+\Delta_{6} \boldsymbol{\gamma} \cdot \hat{\mathbf{k}}+\Delta_{7} \boldsymbol{\gamma} \cdot \hat{\mathbf{k}} \gamma_{5}+\Delta_{8} \gamma_{0},
$$

This result means that $\Delta_{1}, \Delta_{2}, \Delta_{4}$, and $\Delta_{5}$ are gap functions describing condensation of fermion pairs with the same chirality (right-right or left-left), while $\Delta_{3}, \Delta_{6}, \Delta_{7}$, and $\Delta_{8}$ are gap functions describing condensation of fermion pairs with the opposite chirality (right-left and left-right).

Let us summarize the results of this and the preceding subsections. Only particles with the same helicity can condense to form a scalar $(J=0)$ condensate. In the general ansatz $(\vec{B} 2)$, the term 


$$
\Delta_{1} \gamma_{5}+\Delta_{2} \gamma \cdot \hat{\mathbf{k}} \gamma_{0} \gamma_{5}
$$

describes the condensation of fermions with the same chirality in the even-parity channel, while

$$
\Delta_{4}+\Delta_{5} \gamma \cdot \hat{\mathbf{k}} \gamma_{0}
$$

describes condensation of fermions with the same chirality in the odd-parity channel, and

$$
\Delta_{3} \gamma_{0} \gamma_{5}+\Delta_{7} \gamma \cdot \hat{\mathbf{k}} \gamma_{5}
$$

describes condensation of opposite-chirality fermions in the even-parity channel, while

$$
\Delta_{6} \gamma \cdot \hat{\mathbf{k}}+\Delta_{8} \gamma_{0}
$$

describes condensation of opposite-chirality fermions in the odd-parity channel.

\section{Quasiprojector representation of the gap matrix}

The properties of the gap matrix with respect to chirality and helicity suggest a somewhat different representation. Instead of the eight matrices (B1), one can use the eight matrices constructed from chirality, helicity, and energy projectors. We use the following projectors onto states of positive and negative energy for free particles:

$$
\Lambda^{ \pm}(\mathbf{k}) \equiv \frac{E_{\mathbf{k}} \pm\left(\gamma_{0} \boldsymbol{\gamma} \cdot \mathbf{k}+m \gamma_{0}\right)}{2 E_{\mathbf{k}}} \equiv \frac{1 \pm\left(\beta_{\mathbf{k}} \gamma_{0} \boldsymbol{\gamma} \cdot \hat{\mathbf{k}}+\alpha_{\mathbf{k}} \gamma_{0}\right)}{2}
$$

where $\alpha_{\mathbf{k}} \equiv m / E_{\mathbf{k}}, \beta_{\mathbf{k}} \equiv|\mathbf{k}| / E_{\mathbf{k}}$. These differ by $\gamma_{0}$ and the normalization of the spinors from the commonly used projectors [22], but have the advantage that they are regular in the limit $m \rightarrow 0$.

The helicity projectors commute with either the chirality or these energy projectors,

$$
\left[\mathcal{P}_{r, \ell}, \mathcal{P}_{ \pm}(\mathbf{k})\right]=\left[\mathcal{P}_{ \pm}(\mathbf{k}), \Lambda^{ \pm}(\mathbf{k})\right]=0,
$$

but, for finite $m$, as massive spinors are not eigenstates of chirality, the energy projectors do not commute with the chirality projectors,

$$
\left[\mathcal{P}_{r}, \Lambda^{ \pm}(\mathbf{k})\right]=\mp \alpha_{\mathbf{k}} \gamma_{0} \gamma_{5}, \quad\left[\mathcal{P}_{\ell}, \Lambda^{ \pm}(\mathbf{k})\right]= \pm \alpha_{\mathbf{k}} \gamma_{0} \gamma_{5} .
$$

Let us introduce the "quasiprojectors"

$$
\mathcal{P}_{r, \ell \pm}^{ \pm}(\mathbf{k}) \equiv \mathcal{P}_{r, \ell} \mathcal{P}_{ \pm}(\mathbf{k}) \Lambda^{ \pm}(\mathbf{k})
$$

These eight quantities constitute a basis which is equivalent to (B1). Therefore, the general gap matrix (B2) can alternatively be written as

$$
\Delta=\phi_{r+}^{+} \mathcal{P}_{r+}^{+}+\phi_{\ell+}^{+} \mathcal{P}_{\ell+}^{+}+\phi_{r-}^{+} \mathcal{P}_{r-}^{+}+\phi_{\ell-}^{+} \mathcal{P}_{\ell-}^{+}+\phi_{r+}^{-} \mathcal{P}_{r+}^{-}+\phi_{\ell+}^{-} \mathcal{P}_{\ell+}^{-}+\phi_{r-}^{-} \mathcal{P}_{r-}^{-}+\phi_{\ell-}^{-} \mathcal{P}_{\ell-}^{-},
$$

with an obvious notation for the new gap functions $\phi_{r, \ell \pm}^{ \pm}$. The old gap functions $\Delta_{n}$ can be expressed in terms of the new gap functions $\phi$ :

$$
\begin{aligned}
& \Delta_{1}=\frac{1}{8}\left\{\left(1+\beta_{\mathbf{k}}\right)\left[\phi_{r+}^{+}-\phi_{\ell-}^{+}+\phi_{r-}^{-}-\phi_{\ell+}^{-}\right]+\left(1-\beta_{\mathbf{k}}\right)\left[\phi_{r-}^{+}-\phi_{\ell+}^{+}+\phi_{r+}^{-}-\phi_{\ell-}^{-}\right]\right\}, \\
& \Delta_{2}=\frac{1}{8}\left\{\left(1+\beta_{\mathbf{k}}\right)\left[-\phi_{r+}^{+}+\phi_{\ell-}^{+}+\phi_{r-}^{-}-\phi_{\ell+}^{-}\right]+\left(1-\beta_{\mathbf{k}}\right)\left[\phi_{r-}^{+}-\phi_{\ell+}^{+}-\phi_{r+}^{-}+\phi_{\ell-}^{-}\right]\right\}, \\
& \Delta_{3}=\frac{\alpha_{\mathbf{k}}}{8}\left[-\phi_{r+}^{+}-\phi_{r-}^{+}+\phi_{\ell+}^{+}+\phi_{\ell-}^{+}+\phi_{r+}^{-}+\phi_{r-}^{-}-\phi_{\ell+}^{-}-\phi_{\ell-}^{-}\right], \\
& \Delta_{4}=\frac{1}{8}\left\{\left(1+\beta_{\mathbf{k}}\right)\left[\phi_{r+}^{+}+\phi_{\ell-}^{+}+\phi_{r-}^{-}+\phi_{\ell+}^{-}\right]+\left(1-\beta_{\mathbf{k}}\right)\left[\phi_{r-}^{+}+\phi_{\ell+}^{+}+\phi_{r+}^{-}+\phi_{\ell-}^{-}\right]\right\}, \\
& \Delta_{5}=\frac{1}{8}\left\{\left(1+\beta_{\mathbf{k}}\right)\left[-\phi_{r+}^{+}-\phi_{\ell-}^{+}+\phi_{r-}^{-}+\phi_{\ell+}^{-}\right]+\left(1-\beta_{\mathbf{k}}\right)\left[\phi_{r-}^{+}+\phi_{\ell+}^{+}-\phi_{r+}^{-}-\phi_{\ell-}^{-}\right]\right\}, \\
& \Delta_{6}=\frac{\alpha_{\mathbf{k}}}{8}\left[-\phi_{r+}^{+}+\phi_{r-}^{+}+\phi_{\ell+}^{+}-\phi_{\ell-}^{+}+\phi_{r+}^{-}-\phi_{r-}^{-}-\phi_{\ell+}^{-}+\phi_{\ell-}^{-}\right], \\
& \Delta_{7}=\frac{\alpha_{\mathbf{k}}}{8}\left[\phi_{r+}^{+}-\phi_{r-}^{+}+\phi_{\ell+}^{+}-\phi_{\ell-}^{+}-\phi_{r+}^{-}+\phi_{r-}^{-}-\phi_{\ell+}^{-}+\phi_{\ell-}^{-}\right], \\
& \Delta_{8}=\frac{\alpha_{\mathbf{k}}}{8}\left[\phi_{r+}^{+}+\phi_{r-}^{+}+\phi_{\ell+}^{+}+\phi_{\ell-}^{+}-\phi_{r+}^{-}-\phi_{r-}^{-}-\phi_{\ell+}^{-}-\phi_{\ell-}^{-}\right] .
\end{aligned}
$$


The new basis $(\mathrm{B} 25)$ is complete,

$$
\sum_{h=r, \ell} \sum_{s= \pm} \sum_{e= \pm} \mathcal{P}_{h s}^{e}(\mathbf{k})=1
$$

However, using (B24) we find that the quasiprojectors (B25) are not true projectors:

$$
\begin{array}{cl}
\mathcal{P}_{r \pm}^{+} \mathcal{P}_{r \pm}^{+}=\mathcal{P}_{r \pm}^{+}+\frac{\alpha_{\mathbf{k}}}{2} \gamma_{0} \gamma_{5} \mathcal{P}_{\ell \pm}^{+}, & \mathcal{P}_{r \pm}^{+} \mathcal{P}_{\ell \pm}^{+}=-\frac{\alpha_{\mathbf{k}}}{2} \gamma_{0} \gamma_{5} \mathcal{P}_{\ell \pm}^{+}, \\
\mathcal{P}_{\ell \pm}^{+} \mathcal{P}_{r \pm}^{+}=\frac{\alpha_{\mathbf{k}}}{2} \gamma_{0} \gamma_{5} \mathcal{P}_{r \pm}^{+}, & \mathcal{P}_{\ell \pm}^{+} \mathcal{P}_{\ell \pm}^{+}=\mathcal{P}_{\ell \pm}^{+}-\frac{\alpha_{\mathbf{k}}}{2} \gamma_{0} \gamma_{5} \mathcal{P}_{r \pm}^{+}, \\
\mathcal{P}_{r \pm}^{-} \mathcal{P}_{r \pm}^{-}=\mathcal{P}_{r \pm}^{-}-\frac{\alpha_{\mathbf{k}}}{2} \gamma_{0} \gamma_{5} \mathcal{P}_{\ell \pm}^{-}, & \mathcal{P}_{r \pm}^{-} \mathcal{P}_{\ell \pm}^{-}=\frac{\alpha_{\mathbf{k}}}{2} \gamma_{0} \gamma_{5} \mathcal{P}_{\ell \pm}^{-}, \\
\mathcal{P}_{\ell \pm}^{-} \mathcal{P}_{r \pm}^{-}=-\frac{\alpha_{\mathbf{k}}}{2} \gamma_{0} \gamma_{5} \mathcal{P}_{r \pm}^{-}, & \mathcal{P}_{\ell \pm}^{-} \mathcal{P}_{\ell \pm}^{-}=\mathcal{P}_{\ell \pm}^{-}+\frac{\alpha_{\mathbf{k}}}{2} \gamma_{0} \gamma_{5} \mathcal{P}_{r \pm}^{-} \\
\mathcal{P}_{r \pm}^{+} \mathcal{P}_{r \pm}^{-}=\frac{\alpha_{\mathbf{k}}}{2} \gamma_{0} \gamma_{5} \mathcal{P}_{\ell \pm}^{-}, & \mathcal{P}_{r \pm}^{+} \mathcal{P}_{\ell \pm}^{-}=-\frac{\alpha_{\mathbf{k}}}{2} \gamma_{0} \gamma_{5} \mathcal{P}_{\ell \pm}^{-}, \\
\mathcal{P}_{\ell \pm}^{+} \mathcal{P}_{r \pm}^{-}=\frac{\alpha_{\mathbf{k}}}{2} \gamma_{0} \gamma_{5} \mathcal{P}_{r \pm}^{-}, & \mathcal{P}_{\ell \pm}^{+} \mathcal{P}_{\ell \pm}^{-}=-\frac{\alpha_{\mathbf{k}}}{2} \gamma_{0} \gamma_{5} \mathcal{P}_{r \pm}^{-}, \\
\mathcal{P}_{r \pm}^{-} \mathcal{P}_{r \pm}^{+}=-\frac{\alpha_{\mathbf{k}}}{2} \gamma_{0} \gamma_{5} \mathcal{P}_{\ell \pm}^{+}, & \mathcal{P}_{r \pm}^{-} \mathcal{P}_{\ell \pm}^{+}=\frac{\alpha_{\mathbf{k}}}{2} \gamma_{0} \gamma_{5} \mathcal{P}_{\ell \pm}^{+}, \\
\mathcal{P}_{\ell \pm}^{-} \mathcal{P}_{r \pm}^{+}=-\frac{\alpha_{\mathbf{k}}}{2} \gamma_{0} \gamma_{5} \mathcal{P}_{r \pm}^{+}, & \mathcal{P}_{\ell \pm}^{-} \mathcal{P}_{\ell \pm}^{+}=\frac{\alpha_{\mathbf{k}}}{2} \gamma_{0} \gamma_{5} \mathcal{P}_{r \pm}^{+}
\end{array}
$$

(the argument $\mathbf{k}$ was dropped for convenience).

For the remainder of this subsection, we consider the ultrarelativistic limit, $m=\alpha_{\mathbf{k}}=0, \beta_{\mathbf{k}}=1$. In this limit, the quasiprojectors become true projectors. Moreover, four of the eight projectors vanish,

$$
\mathcal{P}_{r-}^{+}=\mathcal{P}_{\ell+}^{+}=\mathcal{P}_{r+}^{-}=\mathcal{P}_{\ell-}^{-}=0 \quad(m=0) .
$$

This is another way of stating that there are no massless right- (left-) handed fermions with negative (positive) helicity and positive energy, and no right- (left-) handed fermions with positive (negative) helicity and negative energy. The reduction in the number of projectors can be intuitively understood noting that massless fermions can be described in terms of 2-component Weyl spinors which require only the simpler algebra of linearly independent $2 \times 2$ matrices.

Another consequence of the ultrarelativistic limit is that one of the three projectors in $(\mathrm{B} 25)$ is redundant:

$$
\begin{aligned}
& \mathcal{P}_{r+, \ell-}^{+}(\mathbf{k}) \equiv \mathcal{P}_{r, \ell} \mathcal{P}_{ \pm}(\mathbf{k}) \equiv \mathcal{P}_{r, \ell} \Lambda^{+}(\mathbf{k}) \equiv \mathcal{P}_{ \pm}(\mathbf{k}) \Lambda^{+}(\mathbf{k}) \\
& \mathcal{P}_{r-, \ell+}^{-}(\mathbf{k}) \equiv \mathcal{P}_{r, \ell} \mathcal{P}_{\mp}(\mathbf{k}) \equiv \mathcal{P}_{r, \ell} \Lambda^{-}(\mathbf{k}) \equiv \mathcal{P}_{\mp}(\mathbf{k}) \Lambda^{-}(\mathbf{k})
\end{aligned}
$$

Thus, we could use any two of the three projectors for chirality, helicity, and energy to construct (B25). We keep all three indices, however, because it facilitates the physical interpretation of our results.

When $m=0$, as the four projectors $(\mathrm{B} 30)$ vanish, the eight independent gap functions reduce to four, and eq. (B26) becomes

$$
\Delta=\phi_{r+}^{+} \mathcal{P}_{r+}^{+}+\phi_{\ell-}^{+} \mathcal{P}_{\ell-}^{+}+\phi_{r-}^{-} \mathcal{P}_{r-}^{-}+\phi_{\ell+}^{-} \mathcal{P}_{\ell+}^{-} .
$$

Using eq. (B27) we obtain for the gap functions $\Delta_{n}$ :

$$
\begin{aligned}
\Delta_{1} & =\frac{1}{4}\left[\phi_{r+}^{+}-\phi_{\ell-}^{+}+\phi_{r-}^{-}-\phi_{\ell+}^{-}\right], \\
\Delta_{2} & =\frac{1}{4}\left[-\phi_{r+}^{+}+\phi_{\ell-}^{+}+\phi_{r-}^{-}-\phi_{\ell+}^{-}\right], \\
\Delta_{4} & =\frac{1}{4}\left[\phi_{r+}^{+}+\phi_{\ell-}^{+}+\phi_{r-}^{-}+\phi_{\ell+}^{-}\right], \\
\Delta_{5} & =\frac{1}{4}\left[-\phi_{r+}^{+}-\phi_{\ell-}^{+}+\phi_{r-}^{-}+\phi_{\ell+}^{-}\right], \\
\Delta_{3} & =\Delta_{6}=\Delta_{7}=\Delta_{8}=0 .
\end{aligned}
$$


The use of the projectors makes it clear that there are only four condensates for massless fermions. This was not apparent previously 3 .

Another consequence of $(\mathrm{B} 32)$ is that the action (B6) decomposes into four parts; with $\psi_{r, \ell \pm}^{ \pm} \equiv \mathcal{P}_{r, \ell \pm}^{ \pm} \psi$,

$$
\begin{aligned}
I[\bar{\psi}, \psi] & =I\left[\bar{\psi}_{r+}^{+}, \psi_{r+}^{+}\right]+I\left[\bar{\psi}_{\ell-}^{+}, \psi_{\ell-}^{+}\right]+I\left[\bar{\psi}_{r-}^{-}, \psi_{r-}^{-}\right]+I\left[\bar{\psi}_{\ell+}^{-}, \psi_{\ell+}^{-}\right] \\
I\left[\bar{\psi}_{h s}^{e}, \psi_{h s}^{e}\right] & \equiv \sum_{k}\left\{\bar{\psi}_{h s}^{e}(k)\left[G_{0}^{+}\right]^{-1}(k) \psi_{h s}^{e}(k)+\frac{1}{2}\left[\bar{\psi}_{C h s}^{e}(k) \Delta(k) \psi_{h s}^{e}(k)+\text { h.c. }\right]\right\}
\end{aligned}
$$

where $h=r, \ell, s= \pm, e= \pm$. We draw the important conclusion that in the ultrarelativistic limit, there is condensation only of fermions with the same helicity and the same chirality.

In the scalar model we find that $\phi_{r+}^{+}=-\phi_{\ell-}^{+}$and $\phi_{r-}^{-}=-\phi_{\ell+}^{-}$; thus

$$
\Delta_{1}=\frac{1}{2}\left[\phi_{r+}^{+}+\phi_{r-}^{-}\right] \quad, \quad \Delta_{2}=\frac{1}{2}\left[-\phi_{r+}^{+}+\phi_{r-}^{-}\right] \quad, \quad \Delta_{4}=\Delta_{5}=0 .
$$

From eqs. (B18), (B19) we conclude that condensation occurs only in the $0^{+}$channel, and not the $0^{-}$channel.

\section{APPENDIX C: RENORMALIZING $\mathcal{F}_{0}^{ \pm}$}

At $T=0$, we rewrite the function $\mathcal{F}_{0}^{ \pm}$as

$$
\mathcal{F}_{0}^{ \pm}=-\frac{g^{2}}{4} \int \frac{\mathrm{d}^{3} \mathbf{q}}{(2 \pi)^{3}}\left[D\left(\mathbf{k}-\mathbf{q}, M_{s}\right) \frac{1}{\epsilon^{ \pm}}-D(\mathbf{k}-\mathbf{q}, 0) \frac{1}{|\mathbf{q}|}\right]+\mathcal{F}_{C T}
$$

with the counter term

$$
\mathcal{F}_{C T} \equiv-\frac{g^{2}}{4} \int \frac{\mathrm{d}^{3} \mathbf{q}}{(2 \pi)^{3}} D(\mathbf{k}-\mathbf{q}, 0) \frac{1}{|\mathbf{q}|}
$$

We assume that the renormalized boson mass vanishes at zero density and temperature, and so in the counter term we take the boson to have zero mass as well.

The first integral in (C1) is now ultraviolet-finite. The counter term (C2) can be computed via dimensional regularization. First rewrite (C2), using 23]

$$
\frac{1}{a^{\alpha} b^{\beta}}=\frac{\Gamma(\alpha+\beta)}{\Gamma(\alpha) \Gamma(\beta)} \int_{0}^{1} \mathrm{~d} x \frac{x^{\alpha-1}(1-x)^{\beta-1}}{[a x+b(1-x)]^{\alpha+\beta}}
$$

and shifting $\mathbf{q} \rightarrow \mathbf{q}+\mathbf{k} x$, into

$$
\mathcal{F}_{C T}=-\frac{g^{2}}{8} \int_{0}^{1} \mathrm{~d} x \frac{1}{\sqrt{1-x}} \int \frac{\mathrm{d}^{3} \mathbf{q}}{(2 \pi)^{3}} \frac{1}{\left[\mathbf{q}^{2}+\mathbf{k}^{2} x(1-x)\right]^{3 / 2}} .
$$

Now compute the last integral in $d=3-\epsilon$ dimensions. Note that this implies $g^{2} \rightarrow g^{2} \tilde{\Lambda}^{3-d}$, where $\tilde{\Lambda}$ has dimensions of energy. The standard formula 23.

$$
\int_{0}^{\infty} \mathrm{d} x x^{\beta} \frac{1}{\left[x^{2}+M^{2}\right]^{\alpha}}=\frac{1}{2} \frac{\Gamma\left(\frac{\beta+1}{2}\right) \Gamma\left(\alpha-\frac{\beta+1}{2}\right)}{\Gamma(\alpha)\left[M^{2}\right]^{\alpha-(\beta+1) / 2}}
$$

leads to

$$
\mathcal{F}_{C T}=-\frac{g^{2}}{16 \pi^{2}}\left[\frac{2}{\epsilon}-\ln \frac{\mathbf{k}^{2}}{\Lambda^{2} e^{2}}\right],
$$

where $\Lambda e$ is the renormalization scale, $\Lambda \equiv \tilde{\Lambda} \sqrt{\pi} e^{1+\gamma / 2}, \gamma$ being the Euler-Mascheroni constant. As usual, the $1 / \epsilon$ term in $(\overline{\mathrm{C}} 6)$ is discarded. 
[1] A.L. Fetter and J.D. Walecka, Quantum Theory of Many-Particle Systems (McGraw-Hill, New York, 1971); A.A. Abrikosov, L.P. Gorkov, and I.E. Dzyaloshinski, Methods of Quantum Field Theory in Statistical Physics (Dover, New York, 1963).

[2] B.C. Barrois, Nucl. Phys. B129, 390 (1977).

[3] D. Bailin and A. Love, Phys. Rep. 107, 325 (1984).

[4] J.F. Donoghue and K.S. Sateesh, Phys. Rev. D 38, 360 (1988); M. Iwasaki and T. Iwado, Phys. Lett. B350, 163 (1995).

[5] L.A. Kondratyuk, M.M. Giannini, and M.I. Krivoruchenko, Phys. Lett. B269, 139 (1991); L.A. Kondratyuk and M.I. Krivoruchenko, Z. Phys. A 344, 99 (1992); T. Schäfer, Phys. Rev. D 57, 3950 (1998).

[6] M. Alford, K. Rajagopal, and F. Wilczek, Phys. Lett. B422, 247 (1998).

[7] R. Rapp, T. Schäfer, E.V. Shuryak, and M. Velkovsky, Phys. Rev. Lett. 81, 53 (1998); T. Schäfer, Nucl. Phys. A642, 45 (1998).

[8] Y. Nambu and G. Jona-Lasinio, Phys. Rev. 122, 345 (1961).

[9] M. Alford, K. Rajagopal, and F. Wilczek, Nucl. Phys. B537, 443 (1999).

[10] A.J. Paterson, Nucl. Phys. B190 [FS3], 188 (1981); R.D. Pisarski and D.L. Stein, J. Phys. A14, 3341 (1981).

[11] R.D. Pisarski and D.H. Rischke, nucl-th/9811104.

[12] T. Schäfer and F. Wilczek, Phys. Rev. Lett. 82, 3956 (1999).

[13] J. Berges and K. Rajagopal, Nucl. Phys. B538, 215 (1999).

[14] K. Langfeld and M. Rho, hep-ph/9811227.

[15] N. Evans, S.D.H. Hsu, and M. Schwetz, Nucl. Phys. B551, 275 (1999); Phys. Lett. B449, 281 (1999).

[16] T. Schäfer and F. Wilczek, Phys. Lett. B450, 325 (1999).

[17] D.T. Son, Phys. Rev. D 59, 094019 (1999).

[18] R.D. Pisarski and D.H. Rischke, in preparation.

[19] M. LeBellac, Thermal Field Theory, (Cambridge University Press, Cambridge, 1996).

[20] V.A. Khodel, V.V. Khodel, and J.W. Clark, Nucl. Phys. A598, 390 (1996).

[21] See, for example: D.H. Rischke and W. Greiner, Int. J. Mod. Phys. E3, 1157 (1994).

[22] F. Gross, Relativistic Quantum Mechanics and Field Theory (Wiley, New York, 1993).

[23] G. t'Hooft and M. Veltman, Nucl. Phys. B44, 189 (1972). 